\title{
The Impact of Decaffeinated Green Tea Extract on Fat Oxidation, Body Composition and Cardio-Metabolic Health in Overweight, Recreationally Active Individuals
}

\author{
Justin D. Roberts ${ }^{1, *(1)}$, Ashley G. B. Willmott ${ }^{1}$, Liam Beasley ${ }^{2}$, Mariette Boal ${ }^{1}$, Rory Davies ${ }^{1}$, Laurence Martin ${ }^{1}$, \\ Havovi Chichger ${ }^{3}$, Lata Gautam $^{3}{ }^{-1}$ and Juan Del Coso ${ }^{4}$ \\ 1 Cambridge Centre for Sport and Exercise Sciences, School of Psychology and Sport Science, Anglia Ruskin \\ University, Cambridge CB1 1PT, UK; ash.willmott@aru.ac.uk (A.G.B.W.); \\ marietteboal.student@gmail.com (M.B.); rorydavies.student@gmail.com (R.D.); \\ lorymartin.student@gmail.com (L.M.) \\ 2 Department for Health, University of Bath, Bath BA2 7AY, UK; lab90@bath.ac.uk \\ 3 School of Life Sciences, Anglia Ruskin University, Cambridge CB1 1PT, UK; \\ havovi.chichger@aru.ac.uk (H.C.); lata.gautam@aru.ac.uk (L.G.) \\ 4 Centre for Sport Studies, Rey Juan Carlos University, 28943 Fuenlabrada, Spain; juan.delcoso@urjc.es \\ * Correspondence: Justin.roberts@aru.ac.uk; Tel.: +44-845-196-5154
}

Citation: Roberts, J.D.; Willmott, A.G.B.; Beasley, L.; Boal, M.; Davies, R.; Martin, L.; Chichger, H.; Gautam, L.; Del Coso, J. The Impact of Decaffeinated Green Tea Extract on Fat Oxidation, Body Composition and Cardio-Metabolic Health in Overweight, Recreationally Active Individuals. Nutrients 2021, 13, 764 https://doi.org/10.3390/nu13030764

Academic Editor: Maria Luz Fernandez

Received: 7 January 2021

Accepted: 24 February 2021

Published: 26 February 2021

Publisher's Note: MDPI stays neutral with regard to jurisdictional claims in published maps and institutional affiliations.

Copyright: (C) 2021 by the authors Licensee MDPI, Basel, Switzerland. This article is an open access article distributed under the terms and conditions of the Creative Commons Attribution (CC BY) license (https:/ / creativecommons.org/licenses/by/ $4.0 /)$.

\begin{abstract}
This study investigated the effect of decaffeinated green tea extract (dGTE), with or without antioxidant nutrients, on fat oxidation, body composition and cardio-metabolic health measures in overweight individuals engaged in regular exercise. Twenty-seven participants ( 20 females, 7 males; body mass: $77.5 \pm 10.5 \mathrm{~kg}$; body mass index: $27.4 \pm 3.0 \mathrm{~kg} \cdot \mathrm{m}^{2}$; peak oxygen uptake $\left(\dot{\mathrm{VO}}_{2 \text { peak }}\right)$ : $\left.30.2 \pm 5.8 \mathrm{~mL} \cdot \mathrm{kg}^{-1} \cdot \mathrm{min}^{-1}\right)$ were randomly assigned, in a double-blinded manner, either: dGTE (400 mg. $\mathrm{d}^{-1}(-)$-epigallocatechin-3-gallate (EGCG), $\left.n=9\right)$; a novel $\mathrm{dGTE}+\left(400 \mathrm{mg} \cdot \mathrm{d}^{-1} \mathrm{EGCG}\right.$, quercetin $\left(50 \mathrm{mg} \cdot \mathrm{d}^{-1}\right.$ ) and $\alpha$-lipoic acid (LA, $\left.\left.150 \mathrm{mg} \cdot \mathrm{d}^{-1}\right), n=9\right)$; or placebo (PL, $\left.n=9\right)$ for 8 weeks, whilst maintaining standardised, aerobic exercise. Fat oxidation ('FAT $\mathrm{MAX}$ ' and steady state exercise protocols), body composition, cardio-metabolic and blood measures (serum glucose, insulin, leptin, adiponectin, glycerol, free fatty acids, total cholesterol, high [HDL-c] and low-density lipoprotein cholesterol [LDL-c], triglycerides, liver enzymes and bilirubin) were assessed at baseline, week 4 and 8. Following 8 weeks of dGTE+, maximal fat oxidation (MFO) significantly improved from $154.4 \pm 20.6$ to $224.6 \pm 23.2 \mathrm{mg} \cdot \mathrm{min}^{-1}(p=0.009)$, along with a $22.5 \%$ increase in the exercise intensity at which fat oxidation was deemed negligible $\left(\mathrm{FAT}_{\mathrm{MIN}} ; 67.6 \pm 3.6 \% \dot{\mathrm{VO}}_{2 \text { peak }}, p=0.003\right)$. Steady state exercise substrate utilisation also improved for dGTE+ only, with respiratory exchange ratio reducing from $0.94 \pm 0.01$ at week 4 , to $0.89 \pm 0.01$ at week $8(p=0.004)$. This corresponded with a significant increase in the contribution of fat to energy expenditure for dGTE+ from $21.0 \pm 4.1 \%$ at week 4 , to $34.6 \pm 4.7 \%$ at week $8(p=0.006)$. LDL-c was also lower (normalised fold change of $-0.09 \pm 0.06$ ) for dGTE + by week 8 ( $p=0.038)$. No other significant effects were found in any group. Eight weeks of dGTE+ improved MFO and substrate utilisation during exercise, and lowered LDL-c. However, body composition and cardio-metabolic markers in healthy, overweight individuals who maintained regular physical activity were largely unaffected by dGTE.
\end{abstract}

Keywords: green tea extract; fat oxidation; body fat; weight loss; exercise

\section{Introduction}

Green tea (GT) polyphenols have been widely investigated for their potential therapeutic health benefits from an antioxidant [1], anti-inflammatory [2,3], chemoprotective [4] and cardio-metabolic perspective [5-7]. The catechin content of GT, of which (-)-epigallocatechin-3-gallate (EGCG) may account for up to $80 \%$ [8], may be primarily related to adaptive mechanisms pertinent to health benefits. GT catechins may have specific thermogenic effects through the inhibition of catechol-o-methyl transferase (COMT) 
and subsequent catecholamine, cyclic adenosine monophosphate (cAMP) and lipolytic activity [9]. Consequently, these effects may enhance whole-body fat oxidation [10,11], and GT catechins may have important metabolic effects pertinent to reduced adipose tissue [12], improved body mass index (BMI) and/or body composition [10], and lowered circulating metabolites (e.g., low-density lipoprotein cholesterol (LDL-c) [13]). However, such mechanisms have also been challenged based on limited evidence from studies [14]. Regular consumption of GT catechins has also been proposed to exert 'calorierestriction-mimetic' effects [15] over time, through modulated cell signalling (e.g., peroxisome proliferator-activated receptor gamma coactivator 1-alpha (PGC1- $\alpha$ ), sirtuin 1 (SIRT1) and AMP-activated protein kinase (AMPK) pathways), influencing gene expression associated with mitochondrial efficiency and fat oxidation [14], and is supported through emerging experimental evidence [16].

In terms of fat oxidation, studies with administration of GT extract (GTE) for $<7$ days, providing between 270 and $375 \mathrm{mg} \cdot \mathrm{d}^{-1}$ EGCG, have reported significant improvements in whole-body fat oxidation rates in untrained and overweight individuals both at rest [17,18], during exercise [18] and post-exercise [19], as well as increased 24 hour energy expenditure [11]. Elevated catecholamine and glycerol concentrations in response to acute EGCG supplementation $[11,19]$ support mechanisms associated with COMT inhibition. However, this may, in part, be explained through the inclusion of high-intensity exercise invoking elevated catecholamine levels [19]. Surprisingly, the benefits observed in these studies occurred despite GTE consumption with food (which has been reported to reduce GTE bioavailability [20]), inferring that observed improvements in fat oxidation likely occurred in response to pre-test fasted intake of GTE. Elsewhere, studies have reported no effect of short-term GTE supplementation on fat oxidation in trained individuals, with doses between 270 and $506 \mathrm{mg} \cdot \mathrm{d}^{-1}$ EGCG [21,22], suggesting that GTE may be beneficial only for untrained/overweight individuals.

In the longer term ( $>3$ weeks), the effects of GTE supplementation on fat oxidation are equivocal. Low-dose GTE supplementation ( $68 \mathrm{mg} \cdot \mathrm{d}^{-1}$ EGCG) for 3 weeks did not improve fat oxidation during exercise in trained individuals $[23,24]$, although improvements in cardio-metabolic and inflammatory metabolites (e.g., increased high-density lipoprotein cholesterol (HDL-c) [23], and reduced C-reactive protein [24]) were reported. A four-week supplementation period of moderate-dose decaffeinated GTE (dGTE; $400 \mathrm{mg} \cdot \mathrm{d}^{-1}$ EGCG) significantly improved total fat oxidation rates during exercise, and reduced body fat percentage in one study [25], but not elsewhere, even when employing higher-dose dGTE (624 mg. $\mathrm{d}^{-1}$ EGCG [26]), with both studies involving healthy, lean males.

With regards to cardio-metabolic risk factors, studies lasting 8-12 weeks also provide equivocal findings. Low-dose GTE supplementation ( $99 \mathrm{mg} \cdot \mathrm{d}^{-1} \mathrm{GTE}$ ) for 8 weeks was not effective in improving body fat percentage, BMI, blood pressure, triglyceride and LDL-c levels over that obtained through introduction of aerobic training in overweight/obese, previously sedentary females [27]. Likewise, high-dose caffeinated GTE $\left(1206.9 \mathrm{mg} \cdot \mathrm{d}^{-1}\right.$ GTE; $595.8 \mathrm{mg} \cdot \mathrm{d}^{-1}$ EGCG) for 12 weeks did not improve resting energy expenditure, body mass, BMI, waist to hip ratio (WHR) or fat mass over that explained through sustained inclusion of a low-energy diet in overweight woman [28]. In contrast, an eight-week GTE supplementation period (500 $\mathrm{mg} \cdot \mathrm{d}^{-1} \mathrm{GTE} ; \sim 225 \mathrm{mg} \cdot \mathrm{d}^{-1}$ EGCG) significantly improved body mass, BMI, body fat percentage, visceral fat area (VFA) and adiponectin levels above that obtained through introduction of aerobic training in previously sedentary overweight women [29] and men [30]. Therefore, it is unclear whether GTE supplementation positively impacts on fat oxidation, body composition and cardio-metabolic risk factors in overweight individuals, particularly when unaccustomed diet or exercise interventions are introduced.

In addition to the range of EGCG doses used, confounding factors such as GTE type and bioavailability may, in part, explain the varying findings observed. Only a handful of studies have employed a dGTE formula $[17,18,25,26]$, whereas positive benefits found elsewhere could be explained through the thermogenic effects of caffeine $[11,19,29,30]$. Whilst positive findings have been observed with a moderate EGCG dose $\left(>300 \mathrm{mg} \cdot \mathrm{d}^{-1}\right)$, 
improving the bioavailability and effectiveness of GTE through standardised consumption away from food [20] and/or inclusion of antioxidant nutrients (e.g., quercetin, $\alpha$-lipoic acid (LA), curcumin [31-34]) may lead to enhanced fat oxidation, body composition, and/or cardio-metabolic risk factor improvement, particularly in overweight individuals. Indeed, precedent for the approach of combining GTE with quercetin or LA has previously been observed in animal models [35,36]. Elsewhere, use of a GT beverage with antioxidant supplementation has been shown to enhance exercise capacity, glucose tolerance and body composition in older individuals [37]. However, there is a paucity of research confirming potential benefits of GTE compounds with human participants, despite novel combinations already being commercially available. Therefore, it is pertinent to investigate the efficacy such compounds compared with GTE only use.

Therefore, this study aimed to assess the longer-term impact of moderate dGTE supplementation (with or without antioxidant nutrients) on fat oxidation, body composition and cardio-metabolic risk factors in overweight, but healthy individuals engaged in regular exercise. It was hypothesised that fat oxidation would be improved with dGTE (particularly when additional antioxidant nutrients are included), resulting in improved body composition and cardio-metabolic risk factors compared with placebo.

\section{Materials and Methods}

\subsection{Ethical Approval and Study Participants}

This study was conducted in accordance with the Declaration of Helsinki (2013), and registered with ClinicalTrials.gov (Identifier: NCT04628624). Ethical approval was obtained from the Faculty of Science and Technology Research Ethics Panel, Anglia Ruskin University (FST/FREP/17/703). Following a priori power calculation assessment (G*power3, Dusseldorf, Germany [38]; using $\alpha=0.05 ; 1-\beta=0.80$; based on observed fat oxidation data $[18,25]$ ), a minimum sample size of 7 (per group) was estimated. A total cohort of 44 participants volunteered for pre-screening selection, from which 13 participants did not meet the study inclusion criteria.

Thirty-one participants were therefore invited to take part in the intervention study. However, 2 participants withdrew at week 4 for personal reasons, and data for 2 participants were excluded based on not meeting supplement adherence or testing criteria. Therefore, a total of 27 participants (20 females, 7 males; mean \pm standard deviation (SD), age: $43 \pm 8$ years; body mass: $77.5 \pm 10.0 \mathrm{~kg}$; height: $1.68 \pm 0.06 \mathrm{~m}$; BMI: $27.4 \pm 3.0 \mathrm{~kg} \cdot \mathrm{m}^{2}$; initial relative peak oxygen uptake $\left.\left(\dot{\mathrm{V}} \mathrm{O}_{2 \text { peak }}\right): 30.2 \pm 5.8 \mathrm{~mL} \cdot \mathrm{kg}^{-1} \cdot \mathrm{min}^{-1}\right)$ satisfactorily completed the intervention, with study power deemed sufficient based on a priori analysis. Participant characteristics for each intervention group are displayed in Table 1. All participants provided written, informed consent, and satisfactorily completed a general health screen prior to study inclusion.

Table 1. Baseline characteristics for intervention groups.

\begin{tabular}{|c|c|c|c|}
\hline Variable & $\begin{array}{c}\text { PL } \\
(n=9 ; \\
4 \text { male, } 5 \text { female })\end{array}$ & $\begin{array}{c}\text { dGTE } \\
(n=9 ; \\
2 \text { male, } 7 \text { female })\end{array}$ & $\begin{array}{c}\mathrm{dGTE}+ \\
(n=9 ; \\
1 \text { male, } 8 \text { female })\end{array}$ \\
\hline Age (years) & $35 \pm 7^{*}$ & $46 \pm 5$ & $47 \pm 5$ \\
\hline Height (m) & $1.71 \pm 0.04$ & $1.66 \pm 0.06$ & $1.67 \pm 0.07$ \\
\hline Body mass $(\mathrm{kg})$ & $82.7 \pm 13.0$ & $74.3 \pm 7.4$ & $75.6 \pm 9.4$ \\
\hline BMI $\left(\mathrm{kg} \cdot \mathrm{m}^{2}\right)$ & $28.2 \pm 4.1$ & $26.8 \pm 2.4$ & $27.1 \pm 2.3$ \\
\hline Body fat (\%) & $32.0 \pm 7.9$ & $33.2 \pm 6.6$ & $36.3 \pm 6.2$ \\
\hline$\dot{\mathrm{VO}}_{2 \text { peak }}\left(\mathrm{L} \cdot \mathrm{min}^{-1}\right)$ & $2.5 \pm 0.7$ & $2.4 \pm 0.6$ & $2.1 \pm 0.5$ \\
\hline$\dot{\mathrm{V}} \mathrm{O}_{2 \text { peak }}\left(\mathrm{mL} \cdot \mathrm{kg}^{-1} \cdot \mathrm{min}^{-1}\right)$ & $30.3 \pm 6.3$ & $32.4 \pm 5.2$ & $27.8 \pm 5.6$ \\
\hline
\end{tabular}

Data presented as the mean \pm SD. PL = placebo; dGTE = decaffeinated green tea extract; dGTE $+=$ decaffeinated green tea extract with antioxidants; BMI = body mass index. No significant differences reported between groups, except age. * denotes significant difference $(p \leq 0.002)$ to dGTE and dGTE+. 


\subsection{Study Design and Eligibility}

This study employed a randomised, repeated-measures, double-blinded, placebocontrolled design over an 8 week period. Following a full study briefing and provision of written, informed consent, volunteers took part in an initial pre-screening assessment with full familiarisation to the main study testing procedures outlined below. Study eligibility required volunteers to: satisfactorily complete a general health screen questionnaire; be over 18 years of age and 'recreationally active' (defined as general exercise activity 1-3 times per week) with a BMI between 25.0 and $29.9 \mathrm{~kg} \cdot \mathrm{m}^{2}$ and/or a body fat percentage in the overweight category for age/gender based on guidelines set out by the American College of Sports Medicine (ACSM) [39]. As a means to further quantify 'recreationally active', participants were required to have a relative $\mathrm{VO}_{2 \text { peak }}$ between 20 and $45 \mathrm{~mL} \cdot \mathrm{kg}^{-1} \cdot \mathrm{min}^{-1}$ during pre-screening testing. Participants with a known history of cardio-metabolic disorders, blood- and liver-related disorders, and recent viral infections were not eligible for study inclusion. Participants were also required to not be consuming other nutritional or thermogenic supplements (e.g., GTE, creatine, weight loss products) or medication for at least 4 weeks prior to beginning the study intervention.

\subsection{Laboratory Procedures}

All testing procedures were carried out in the Cambridge Centre for Sport and Exercise Sciences, Human Physiology Laboratory, Anglia Ruskin University under controlled environmental conditions $\left(\mathrm{M} \pm \mathrm{SD}\right.$; temperature: $18.5 \pm 1.3{ }^{\circ} \mathrm{C}$; barometric pressure: $1009.4 \pm 12.2 \mathrm{mBar}$; and relative humidity: $44.7 \pm 7.8 \%$ ). In addition to initial pre-screening, participants visited the laboratory at baseline (week 0 ), and at the end of weeks 4 and 8 of the intervention period. All testing was undertaken in the morning, with participants being tested at the same time of day across the intervention. In the week leading into each laboratory visit, participants were required to complete dietary intake and activity diaries (see Section 2.5). In addition, as a means to standardise each pre-testing period, in the $24 \mathrm{~h}$ prior, participants were required to rest/no exercise, consume a standardised evening meal ( 10 kcal $\cdot \mathrm{kg}^{-1}$, of which $50 \%$ carbohydrate, $20 \%$ protein, $30 \%$ fat), and arrive in a euhydrated, fasted state ( 10 hours) having refrained from consuming caffeinated products prior to testing.

Upon arrival, participants rested for $\sim 10 \mathrm{~min}$ in a supine position prior to duplicate assessment of resting heart rate and blood pressure (Omron 705CP, Kyoto, Japan). Following this, a venous whole blood sample was collected from participants by a qualified phlebotomist into triplicate $4 \mathrm{~mL}$ serum clot activator Vacuette ${ }^{\mathrm{TM}}$ tubes (Greiner Bio-One $\mathrm{GmbH}$, Kremsmunster, Austria). Samples were centrifuged for $10 \mathrm{~min}$ at $2000 \mathrm{rcf}$ according to manufacturer recommendations, with aliquotted serum pipetted into sterile, nonpyrogenic, polypropylene cryovials (Fisherbrand, Fisher Scientific, Loughborough, UK) and frozen at $-80{ }^{\circ} \mathrm{C}$ for later assessment of blood analytes (see Section 2.6).

\subsubsection{Anthropometric Measures}

Participants then underwent body composition assessment, with height measured using a stadiometer (Seca CE123, Hamburg, Germany). Body mass and body fat percentage were assessed through the use of bioelectrical impedance analysis scales (Tanita SC-330ST, Amsterdam, The Netherlands). Body fat percentage was additionally confirmed using an 8-site skinfold calliper assessment (following guidelines outlined by the International Society for the Advancement of Kinanthropometry (ISAK)). Specific girth measures were undertaken for abdomen, thigh and calf locations, as well as waist to hip ratio (WHR) conforming to ISAK/ACSM guidelines. Sagittal abdominal diameter (SAD) was also measured using a Holtain-Kahn abdominal caliper (model 609, Holtain Ltd., Crosswell, Pembrokeshire, UK), with visceral fat area (VFA) estimated from validated equations [40]. Anthropometric measures were undertaken in triplicate by the same researcher across the study period, with average readings for each measure used for analyses. 


\subsubsection{Incremental Exercise Fat Oxidation Assessment}

Participants were fitted with a Polar FS2 telemetric heart rate (HR) monitor (Polar Electro Ltd., Kempele, Finland) and a suitable face-mask for expired air assessment (Hans Rudolph 7450, Cranlea Human Performance Ltd., Bournville, Birmingham, UK) prior to an initial resting period. Resting expired air was then measured using a Metalyser 3B automated gas-analyser (Cortex Biophysik, Leipzig, Germany), after which participants underwent a maximal fat oxidation ('FAT $\mathrm{MAX}_{\mathrm{X}}$ ) test using a Lode Excalibur sport cycle-

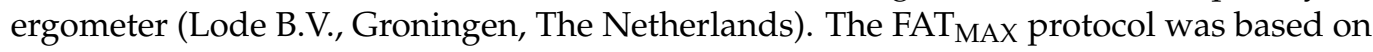
original research [41], but modified in line with recent studies involving similar cohorts to the present study [42] and pilot work assessment.

Briefly, participants completed a graded exercise test to volitional exhaustion, starting at $30 \mathrm{~W}$ (acting as a warm-up stage) with $20 \mathrm{~W}$ increments every $3 \mathrm{~min}$. Cadence was maintained between 75 and $85 \mathrm{rpm}$ throughout. Breath-to-breath expired air analysis was undertaken throughout the test, along with telemetric HR and self-assessed rating of perceived exertion (RPE) [43] in the last minute of each stage. Maximal individual effort was determined when two of the following criteria were met: (i) a change in oxygen consumption $\left(\mathrm{V}_{2}\right)$ of $<2 \mathrm{~mL} \cdot \mathrm{kg}^{-1} \cdot \mathrm{min}^{-1}$ with increasing workload; (ii) attainment of $95 \%$ of age-predicted $\mathrm{HR}_{\max }$ (220-age); (iii) a respiratory exchange ratio (RER) > 1.05; and (iv) an RPE >9 (0-10 scale) along with evidence of volitional fatigue symptoms. $\dot{V O}_{2 \text { peak }}$ was estimated from the highest $30 \mathrm{sec}$ moving average oxygen consumption.

Fat oxidation rates $\left(\mathrm{FAT}_{\mathrm{OX}} ; \mathrm{mg} \cdot \mathrm{min}^{-1}\right.$ ) were derived from $\mathrm{V}_{2}$ and carbon dioxide $\left(\mathrm{VCO}_{2}\right)$ expired air assessment $\left(\mathrm{L} \cdot \mathrm{min}^{-1}\right)$ using stoichiometric equations $[18,44]$, with protein oxidation assumed negligible, as follows:

$$
\operatorname{FAT}_{\mathrm{OX}}=\left(1.695 \times \dot{\mathrm{VO}_{2}}\right)-(1.701 \times \dot{\mathrm{V} C O})_{2},
$$

Using individual $\mathrm{FAT}_{\mathrm{Ox}}$ data for each stage plotted against exercise intensity (\% $\dot{\mathrm{V}}_{2 \text { peak }}$ ), a 2nd-order polynomial curve was fitted from which maximal fat oxidation rate $\left(\mathrm{MFO} ; \mathrm{mg} \cdot \mathrm{min}^{-1}\right)$, maximal fat oxidation exercise intensity $\left(\mathrm{FAT}_{\mathrm{MAX}}\right.$, defined as $\% \dot{\mathrm{V}}_{2 \text { peak }}$ at which MFO occurred) and minimum fat oxidation exercise intensity (FAT MIN $_{\text {, }}$ defined as the $\% \dot{\mathrm{VO}}_{2 \text { peak }}$ where $\mathrm{FAT}_{\mathrm{OX}}$ was deemed zero) were derived, based on previous research [45].

\subsubsection{Steady State Exercise Assessment}

Following a standardised 30 min rest period (with resting $\dot{\mathrm{VO}}_{2}$ and $\mathrm{HR}$ comparable to pre-exercise conditions), participants underwent a fixed-intensity exercise test (60\% of individual $\left.\dot{\mathrm{VO}}_{2 \text { peak }}\right)$, using the same cycle-ergometer, with cadence maintained between 75 and $85 \mathrm{rpm}$ for $60 \mathrm{~min}$. Perceived exertion and HR were measured at $5 \mathrm{~min}$ and each 15 min thereafter. Breath-to-breath expired air was continuously measured throughout the submaximal exercise period, with $60 \mathrm{sec}$ average data recorded upon test completion. Fat oxidation rates were derived from the previous stoichiometric Equation (1). Carbohydrate oxidation rates $\left(\mathrm{CHO}_{\mathrm{Ox}} ; \mathrm{mg} \cdot \mathrm{min}^{-1}\right)$ and energy expenditure $\left(\mathrm{EE} ; \mathrm{kcal} \cdot \mathrm{min}^{-1}\right)$ were derived from the following equations [44]:

$$
\begin{gathered}
\mathrm{CHO}_{\mathrm{OX}}=\left(4.210 \times \dot{\mathrm{VCO}_{2}}\right)-\left(2.962 \times \dot{\mathrm{VO}_{2}}\right), \\
\mathrm{EE}=\left(0.550 \times \dot{\mathrm{V} C O}{ }_{2}\right)+\left(4.471 \times \dot{\mathrm{VO}_{2}}\right),
\end{gathered}
$$

Additionally, both fat and carbohydrate oxidation stability (expressed as a \%) were assessed on the basis of the duration maintained within $\pm 10 \%$ of mean $\mathrm{FAT}_{\mathrm{OX}}$ and $\mathrm{CHO}_{\mathrm{OX}}$ rates, respectively. Contributions of both carbohydrate and fat to EE were calculated on the 
basis of caloric conversion of estimated total $\mathrm{CHO}_{\mathrm{Ox}}$ and $\mathrm{FAT}_{\mathrm{OX}}\left(\mathrm{g} \cdot \mathrm{h}^{-1}\right)$ expressed as a percentage of EE $\left(\mathrm{kcal} \cdot \mathrm{h}^{-1}\right)$.

\subsection{Nutritional Interventions}

Following baseline assessment, participants were allocated, in a double-blinded manner, to one of three intervention groups using a random number generator. Participants were randomly assigned to an 8 week intervention ( 2 hydroxypropyl methyl cellulose capsules daily, split dose taken away from food) of either: (i) placebo (PL; containing a total of $892 \mathrm{mg} \cdot \mathrm{d}^{-1}$ potato fibre as a bulking agent); (ii) decaffeinated green tea extract (dGTE; $580 \mathrm{mg} \cdot \mathrm{d}^{-1} \mathrm{dGTE}, 70 \%$ standardised, delivering $400 \mathrm{mg} \cdot \mathrm{d}^{-1} \mathrm{EGCG}$, matched weighted with additional bulking agent, negligible caffeine trace $(\leq 0.5 \%)$ ); or (iii) a novel decaffeinated green tea extract formula $\left(\mathrm{dGTE}+; 580 \mathrm{mg} \cdot \mathrm{d}^{-1} \mathrm{dGTE}\left(400 \mathrm{mg} \cdot \mathrm{d}^{-1}\right.\right.$ EGCG, negligible caffeine trace $\leq 0.5 \%$ ), with quercetin $\left(50 \mathrm{mg} \cdot \mathrm{d}^{-1}\right)$ and $\alpha$-lipoic acid (LA; $150 \mathrm{mg} \cdot \mathrm{d}^{-1}$ ), also matched weighted with additional bulking agent).

All supplementation was supplied and pre-capsulated by Biocare Ltd. (Redditch, Birmingham, UK), and verified through Nutraceuticals Group Europe (Merstham, Surrey, UK) with dGTE sourced from Changsha Active Ingredients Group Inc. (Changsha, China), based on our previous research [25]. All supplements were provided in standardised opaque sealed pots for hygiene and double-blinding purposes. To monitor supplement adherence, participants completed a daily compliance record throughout the intervention. As a further measure, participants were initially provided a 4 week supplement supply and returned pots at the first follow-up session, with excess capsules counted. Participants were then provided with a second 4 week supply, with pots returned and checked at the final testing session, and overall adherence levels cross-referenced against compliance records. A threshold target of $90 \%$ adherence was used for protocol compliance.

\subsection{Dietary Intake and Exercise Monitoring}

Following the pre-screening session, participants included in the main intervention completed a 7 day food diary using a smart phone application (MyFitnessPal), validated in previous research [46,47]. This served partly as a familiarisation process, and also to gain awareness of typical individual dietary intakes. Throughout this study, participants were requested to maintain typical dietary patterns for consistency. Furthermore, in the 7 day period leading into each laboratory session, participants completed a habitual record of dietary intake to assess for consistency. Individual guidance was provided in diary collation, with emphasis on meal content, portion size and weight, and fluid intake. Diaries were assessed using Nutritics Professional Dietary Analysis software (Nutritics Ltd., Co. Dublin, Ireland) by the same researcher. In addition, individual food items were cross referenced against the U.S. Department of Agriculture (USDA) database on the flavonoid content of selected foods, and the Phenol-Explorer database as a means to estimate dietary quercetin intake.

In conjunction with this, participants were also provided with a standard activity $\log$ and HR monitor, and requested to track all exercise activity prior to the start of the intervention (acting as habitual reference), and each week across the 8 week study period. Participants recorded exercise activity, mean session HR, duration and overall session rating of perceived exertion (sRPE) from which training load, monotony and strain were determined as previously described $[48,49]$. To standardise weekly physical activity, and aligned with participants' habitual exercise patterns, following the baseline laboratory session, participants were provided with general exercise guidance to be maintained throughout this study. This involved targeting $\sim 150 \mathrm{~min}$ of moderate aerobic exercise $\left(\sim 3-5\right.$ sessions $\left.\cdot \mathrm{wk}^{-1}\right)$, at $\sim 60-65 \% \dot{\mathrm{V}} \mathrm{O}_{2 \text { peak }}\left(\sim 75-80 \% \mathrm{HR}_{\max }\right)$ in accordance with guidelines set out by the American College of Sports Medicine [39,50]. 


\subsection{Biochemical Assays}

All blood samples were analysed independently in conjunction with the NIHR Cambridge Biomedical Research Centre, Core Biochemical Analysis Laboratory (CBAL), Addenbrookes Hospital, Cambridge. Serum samples were assessed at week 0, 4 and 8 using a Siemens Dimension EXL analyser (Siemens Healthcare Ltd., Camberley, Surrey, UK) for the following analytes (between-batch imprecision (BBI) included): glucose (BBI: $3.6 \%$ at $3.5 \mathrm{mmol} \cdot \mathrm{L}^{-1}$ ); triglycerides (Tg; $\mathrm{BBI}: 5.5 \%$ at $0.9 \mathrm{mmol} \cdot \mathrm{L}^{-1}, 3.4 \%$ at $2.4 \mathrm{mmol} \cdot \mathrm{L}^{-1}$ ); total cholesterol (Tc; BBI: $4.9 \%$ at $2.5 \mathrm{mmol} \cdot \mathrm{L}^{-1}, 2.0 \%$ at $6.2 \mathrm{mmol} \cdot \mathrm{L}^{-1}$ ); and HDL-c (BBI: $4.6 \%$ at $0.9 \mathrm{mmol} \cdot \mathrm{L}^{-1}, 3.6 \%$ at $2.3 \mathrm{mmol} \cdot \mathrm{L}^{-1}$ ), with LDL-c (BBI: $3.9 \%$ at $1.4 \mathrm{mmol} \cdot \mathrm{L}^{-1}$, $5.0 \%$ at $3.2 \mathrm{mmol} \cdot \mathrm{L}^{-1}$ ) derived from the Friedewald equation [51] as follows:

$$
\text { LDL-c }=\text { Tc }- \text { HDL-c }-(\mathrm{Tg} / 2.2),
$$

Additionally, serum insulin was assessed using a sandwich chemiluminescence immunoassay via a DiaSorin Liaison XL analyser (DiaSorin, Saluggia (VC), Italy), with BBI noted as $11.0 \%$ at $34.0 \mathrm{pmol} \cdot \mathrm{L}^{-1}$ and $7.0 \%$ at $135.0 \mathrm{pmol} \cdot \mathrm{L}^{-1}$. An updated Homeostasis Model Assessment (HOMA) was employed to estimate steady state beta cell function (\%B), insulin sensitivity $(\% \mathrm{~S})$ and insulin resistance (IR) using a HOMA2 calculator (v2.2.3., Diabetes Trials Unit, University of Oxford, UK) based on previous research [52]. Serum glycerol was quantified using a coupled enzyme assay involving glycerol kinase and glycerol phosphate oxidase (Sigma-Aldrich, Merck Life Science UK Ltd., Gillingham, Dorset, UK; no BBI quoted). Based on the enzymatic conversion of free fatty acids (FFA) to acyl CoA by acyl-Co A synthetase, FFA were determined using a Roche half-micro test assay kit (Sigma-Aldrich, Merck Life Science UK Ltd., Gillingham, Dorset, UK; BBI: $12.5 \%$ at $122 \mu \mathrm{mol} \cdot \mathrm{L}^{-1}, 4.5 \%$ at $\left.466 \mu \mathrm{mol} \cdot \mathrm{L}^{-1}\right)$. Serum leptin and adiponectin were assessed using an AutoDELFIA analyser (Perkin Elmer LAS (UK) Ltd., Beaconsfield, Buckinghamshire, UK), employing in-house two-site microtiter plate-based DELFIA assay (BBI for leptin: $7.1 \%$ at $2.7 \mathrm{ng} \cdot \mathrm{mL}^{-1}$, $3.9 \%$ at $14.9 \mu \mathrm{g} \cdot \mathrm{mL}^{-1}$ and $5.7 \%$ at $54.9 \mu \mathrm{g} \cdot \mathrm{mL}^{-1}$; BBI for adiponectin: $5.4 \%$ at $3.6 \mu \mathrm{g} \cdot \mathrm{mL}^{-1}$, $5.2 \%$ at $9.2 \mu \mathrm{g} \cdot \mathrm{mL}^{-1}$ and $5.8 \%$ at $15.5 \mu \mathrm{g} \cdot \mathrm{mL}^{-1}$ ), with antibodies and standards supplied by R\&D systems (R\&D Systems Europe, BioTechne, Abingdon, UK).

An important feature of the current study, and with recent concerns around potential for high-dose catechin-related hepatic toxicity, a liver enzyme panel was also carried out using a Siemens Dimension EXL analyser (Siemens Healthcare Ltd., Camberley, Surrey, UK) for the following analytes: alkaline phosphatase (ALP; BBI: $16.3 \%$ at $40 \mathrm{U} \cdot \mathrm{L}^{-1}, 6.4 \%$ at $282 \mathrm{U} \cdot \mathrm{L}^{-1}$ ); alanine aminotransferase (ALT; BBI: $8.1 \%$ at $22 \mathrm{U} \cdot \mathrm{L}^{-1}, 3.6 \%$ at $180 \mathrm{U} \cdot \mathrm{L}^{-1}$ ); aspartate aminotransferase (AST; BBI: $5.0 \%$ at $38 \mathrm{U} \cdot \mathrm{L}^{-1}, 2.5 \%$ at $253 \mathrm{U} \cdot \mathrm{L}^{-1}$ ); and bilirubin (BBI: $2.7 \%$ at $14 \mu \mathrm{mol} \cdot \mathrm{L}^{-1}, 1.0 \%$ at $74 \mu \mathrm{mol} \cdot \mathrm{L}^{-1}$ ). As a means to detect any acute elevations in liver enzymes, participants also had an additional blood sample collected at week 2 of the intervention as a precautionary measure.

\subsection{Statistical Analyses}

Statistical analyses were performed using SPSS (IBM, Version 26.0). Normality of data was verified by the Shapiro-Wilk test. Outliers were identified by inspection of box plots $>1.5$ interquartile range in SPSS. Baseline measures were assessed using a between-groups analysis of variance (ANOVA), or where applicable (e.g., dietary analyses) an independent samples t-test was employed. A mixed-design repeated-measures ANOVA (group, time) were performed for main analyses, with Bonferroni post-hoc comparisons where applicable. Where sphericity was violated a Greenhouse-Geisser correction was applied. An alpha level of $\leq 0.05$ was employed for statistical significance, with effect size (partial eta squared; $\eta_{\mathrm{p}}{ }^{2}$ ) also reported (small $=0.02$, medium $=0.13$, large $=0.26$ ). Data are reported as the mean \pm SE. 


\section{Results}

\subsection{Dietary Intake, Supplement Adherence and Exercise Monitoring}

Dietary analysis comparisons across the intervention are shown in Table 2. No significant differences were reported between or within groups across the 8 week intervention period for energy, carbohydrate, fat or protein intake $(p>0.05)$. In addition, no differences were reported between mean energy intake compared with expected maintenance caloric intake $(p>0.05)$ overall or within groups at week 4 or 8 , demonstrating dietary consistency.

Table 2. Mean relative energy and macronutrient intake comparisons between groups.

\begin{tabular}{|c|c|c|c|}
\hline Variable & PL & dGTE & dGTE+ \\
\hline \multicolumn{4}{|c|}{ 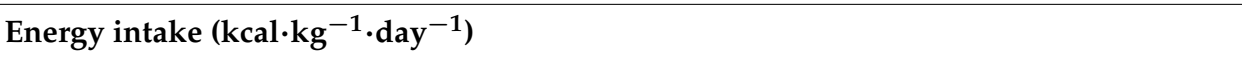 } \\
\hline Baseline & $23.7 \pm 1.2$ & $25.8 \pm 1.4$ & $25.4 \pm 1.3$ \\
\hline Week 4 & $24.8 \pm 1.8$ & $25.4 \pm 1.6$ & $26.1 \pm 1.7$ \\
\hline Week 8 & $24.4 \pm 1.5$ & $25.5 \pm 1.3$ & $25.6 \pm 1.6$ \\
\hline \multicolumn{4}{|c|}{ Fat $\left(\mathrm{g} \cdot \mathrm{kg}^{-1} \cdot \mathrm{day}^{-1}\right)$} \\
\hline Baseline & $0.9 \pm 0.1$ & $0.9 \pm 0.1$ & $1.0 \pm 0.1$ \\
\hline Week 4 & $1.0 \pm 0.1$ & $0.8 \pm 0.1$ & $1.0 \pm 0.1$ \\
\hline Week 8 & $0.9 \pm 0.1$ & $0.9 \pm 0.1$ & $1.0 \pm 0.1$ \\
\hline \multicolumn{4}{|c|}{ Carbohydrate $\left(\mathrm{g} \cdot \mathrm{kg}^{-1} \cdot \mathrm{day}^{-1}\right)$} \\
\hline Baseline & $2.8 \pm 0.2$ & $3.0 \pm 0.2$ & $2.7 \pm 0.2$ \\
\hline Week 4 & $2.7 \pm 0.3$ & $3.1 \pm 0.2$ & $2.8 \pm 0.3$ \\
\hline Week 8 & $2.7 \pm 0.2$ & $3.0 \pm 0.2$ & $2.7 \pm 0.2$ \\
\hline \multicolumn{4}{|c|}{ Protein $\left(\mathrm{g} \cdot \mathrm{kg}^{-1} \cdot \mathrm{day}^{-1}\right)$} \\
\hline Baseline & $0.9 \pm 0.0$ & $1.2 \pm 0.1$ & $1.1 \pm 0.1$ \\
\hline Week 4 & $0.9 \pm 0.1$ & $1.2 \pm 0.2$ & $1.1 \pm 0.1$ \\
\hline Week 8 & $1.0 \pm 0.1$ & $1.1 \pm 0.1$ & $1.1 \pm 0.1$ \\
\hline
\end{tabular}

Data represent average daily intake. $\mathrm{PL}=$ placebo; $\mathrm{dGTE}=$ decaffeinated green tea extract; $\mathrm{dGTE}+=$ decaffeinated green tea extract with antioxidants. No significant differences reported between or within groups $(p>0.05)$.

Across the 8 week intervention, average daily energy intake for dGTE+ was $1918 \pm 66 \mathrm{kcal} \cdot \mathrm{d}^{-1}$ (or $25.7 \pm 1.2 \mathrm{kcal} \cdot \mathrm{kg}^{-1} \cdot$ day $^{-1}$ ) compared with $1882 \pm 96 \mathrm{kcal} \cdot \mathrm{d}^{-1}$ (or $25.6 \pm 1.2 \mathrm{kcal} \cdot \mathrm{kg}^{-1} \cdot \mathrm{day}^{-1}$ ) for dGTE, and $1975 \pm 96 \mathrm{kcal} \cdot \mathrm{d}^{-1}$ (or $24.3 \pm 1.5 \mathrm{kcal} \cdot \mathrm{kg}^{-1} \cdot \mathrm{day}^{-1}$ ) for PL. Macronutrient intake was also comparable, with an average fat intake of $1.0 \pm$ $0.1 \mathrm{~g} \cdot \mathrm{kg}^{-1} \cdot \mathrm{day}^{-1}$ for dGTE+ compared $0.9 \pm 0.1 \mathrm{~g} \cdot \mathrm{kg}^{-1} \cdot \mathrm{day}^{-1}$ for dGTE and $0.9 \pm$ $0.1 \mathrm{~g} \cdot \mathrm{kg}^{-1} \cdot \mathrm{day}^{-1}$ for PL. Likewise, average carbohydrate intake was $2.7 \pm 0.2 \mathrm{~g} \cdot \mathrm{kg}^{-1} \cdot \mathrm{day}^{-1}$ for dGTE+ compared with $3.0 \pm 0.2 \mathrm{~g} \cdot \mathrm{kg}^{-1} \cdot \mathrm{day}^{-1}$ for dGTE and $2.7 \pm 0.2 \mathrm{~g} \cdot \mathrm{kg}^{-1} \cdot \mathrm{day}^{-1}$ for PL. Similarly, average protein intake was comparable between groups at $1.1 \pm$ $0.1 \mathrm{~g} \cdot \mathrm{kg}^{-1} \cdot \mathrm{day}^{-1}$ for dGTE,$+ 1.2 \pm 0.1 \mathrm{~g} \cdot \mathrm{kg}^{-1} \cdot \mathrm{day}^{-1}$ for dGTE, and $0.9 \pm 0.0 \mathrm{~g} \cdot \mathrm{kg}^{-1} \cdot \mathrm{day}^{-1}$ for PL.

Estimation of dietary quercetin highlighted a mean overall intake of $20.6 \pm 8.5 \mathrm{mg} \cdot \mathrm{d}^{-1}$, with no differences reported between groups $\left(20.1 \pm 7.3 \mathrm{mg} \cdot \mathrm{d}^{-1}\right.$ for PL; $20.0 \pm 8.8 \mathrm{mg} \cdot \mathrm{d}^{-1}$ for dGTE; and $21.8 \pm 10.4 \mathrm{mg} \cdot \mathrm{d}^{-1}$ for dGTE,$+ p>0.05$ ). Mean adherence to the supplement intervention was $95.4 \pm 1.0 \%$. Within groups, adherence rates were $93.0 \pm 1.1 \%, 96.3 \pm$ $0.8 \%$ and $96.9 \pm 1.3 \%$ for PL, dGTE and dGTE+, respectively, with a significant difference reported between PL and dGTE+ $(p=0.04)$ only.

Exercise monitoring training load comparisons are shown in Table 3. No significant differences were reported between groups across the intervention for training load, monotony or strain $(p>0.05)$ demonstrating relative consistency. Mean weekly duration was reported at $189.7 \pm 22.4 \mathrm{~min}, 204.0 \pm 20.9 \mathrm{~min}$ and $196.4 \pm 29.1 \mathrm{~min}$ for PL, dGTE and dGTE+, respectively $(p>0.05)$, meeting expected recommendations. No significant differences were observed for mean HR compared to training recommendations $\left(75-80 \% \mathrm{HR}_{\max }\right)$ for $\mathrm{PL}\left(82 \pm 2 \% \mathrm{HR}_{\max }\right)$, $\mathrm{dGTE}\left(79 \pm 2 \% \mathrm{HR}_{\max }\right)$ and dGTE $+\left(79 \pm 1 \% \mathrm{HR}_{\max }\right)$, respectively $(p>0.05)$. 
Table 3. Estimated mean training load, monotony and strain between intervention groups prior to and across the experimental period.

\begin{tabular}{lccc}
\hline Variable & PL & dGTE & dGTE+ \\
\hline Weekly training load (AU) & & & \\
Baseline & $869 \pm 129$ & $1134 \pm 136$ & $848 \pm 144$ \\
Week 4 & $996 \pm 139$ & $893 \pm 127$ & $918 \pm 96$ \\
Week 8 & $888 \pm 166$ & & $795 \pm 141$ \\
\hline Training monotony (AU) & & & \\
Baseline & $0.85 \pm 0.08$ & $0.92 \pm 0.12$ & $0.90 \pm 0.07$ \\
Week 4 & $0.82 \pm 0.05$ & $0.92 \pm 0.07$ & $1.02 \pm 0.09$ \\
Week 8 & $0.78 \pm 0.02$ & & $0.98 \pm 0.16$ \\
\hline Training strain (AU) & & $1090 \pm 205$ & $819 \pm 223$ \\
Baseline & $764 \pm 139$ & $824 \pm 143$ & $1070 \pm 188$ \\
Week 4 & $787 \pm 88$ & $804 \pm 137$ & $969 \pm 350$ \\
Week 8 & $666 \pm 116$ & & \\
\hline
\end{tabular}

Data represent arbitrary units (AU). PL = placebo; dGTE = decaffeinated green tea extract; dGTE $+=$ decaffeinated green tea extract with antioxidants. No significant differences reported between or within groups $(p>0.05)$.

\subsection{Fat Oxidation Data}

Mean fat oxidation measures are shown in Table 4, with normalised fold change shown in Figure 1. No significant differences were reported at baseline for all variables $(p>0.05)$. A significant interaction effect was found for maximal fat oxidation (MFO; $\left.\mathrm{F}=3.41, p=0.016, \eta_{\mathrm{p}}{ }^{2}=0.22\right)$, with post-hoc analysis highlighting a $45.4 \%$ increase in MFO for dGTE + at week $8(p=0.009)$ compared to baseline only. This was supported with a noted trend at week 4 for dGTE $+(p=0.063)$ also compared to baseline. Similarly, dGTE+ resulted in $49.4 \%$ increase in relative MFO (interaction effect; $\mathrm{F}=3.67, p=0.011$, $\left.\eta_{\mathrm{p}}{ }^{2}=0.23\right)$ by week 8 compared to baseline $(p=0.024)$, with a trend noted compared to week $4(p=0.059)$. dGTE had a positive, but non-significant impact on MFO $(+12.7 \%)$ and MFO $\cdot \mathrm{FFM}^{-1}(+13.9 \%)$, whereas PL resulted in a non-significant reduction in MFO $(-11.8 \%)$ and MFO. $\mathrm{FFM}^{-1}(-21.4 \% ; p>0.05)$.

No significant effects were found for $\mathrm{FAT}_{\mathrm{MAX}}$ or $\mathrm{HR}$ at $\mathrm{FAT}_{\mathrm{MAX}}$ within or between groups $(p>0.05)$. However, a positive interaction effect was observed for $\mathrm{FAT}_{\mathrm{MIN}}(\mathrm{F}=6.02$, $p=0.001, \eta_{\mathrm{p}}{ }^{2}=0.33$ ), with dGTE+ resulting in a $22.5 \%$ increase in $\mathrm{FAT}_{\text {MIN }}$ by week 8 compared with week $4(p=0.003)$ and a trend noted compared to baseline $(p=0.056)$. When expressed as normalised fold change, a significant effect was found for FAT $_{\text {MIN }}$ by week $8\left(\mathrm{~F}=4.77, p=0.018, \eta_{\mathrm{p}}{ }^{2}=0.28\right)$, with dGTE+ demonstrating a $+0.18 \pm 0.08$ fold change compared with PL $(-0.08 \pm 0.05 ; p=0.023)$. Throughout the intervention, as expected, $\dot{\mathrm{V}} \mathrm{O}_{2 \text { peak }}$ was maintained for PL: $2.5 \pm 0.2 \mathrm{~L} \cdot \mathrm{min}^{-1}, 2.4 \pm 0.1 \mathrm{~L} \cdot \mathrm{min}^{-1}$ and $2.4 \pm 0.1 \mathrm{~L} \cdot \mathrm{min}^{-1}$; dGTE: $2.4 \pm 0.2 \mathrm{~L} \cdot \mathrm{min}^{-1}, 2.5 \pm 0.2 \mathrm{~L} \cdot \mathrm{min}^{-1}$, and $2.4 \pm 0.2 \mathrm{~L} \cdot \mathrm{min}^{-1}$; and dGTE+: $2.1 \pm 0.2 \mathrm{~L} \cdot \mathrm{min}^{-1}, 2.3 \pm 0.2 \mathrm{~L} \cdot \mathrm{min}^{-1}$, and $2.1 \pm 0.2 \mathrm{~L} \cdot \mathrm{min}^{-1}$, with no differences reported within or between groups at week 0,4 and 8 , respectively $(p>0.05)$.

\subsection{Steady State Exercise Data}

Mean steady state exercise measures are shown in Table 5, with normalised fat oxidation data shown in Figure 2. Mean exercise intensity during laboratory testing was comparable across the intervention (baseline: $60.2 \pm 1.4 \% \dot{\mathrm{VO}}_{2 \text { peak }}, \dot{\mathrm{V}} \mathrm{O}_{2}: 1.4 \pm 0.1 \mathrm{~L} \cdot \mathrm{min}^{-1}$; week 4: $59.8 \pm 1.6 \% \dot{\mathrm{VO}}_{2 \text { peak }}, \dot{\mathrm{VO}}_{2}: 1.4 \pm 0.1 \mathrm{~L} \cdot \mathrm{min}^{-1}$; week 8: $58.7 \pm 1.5 \% \dot{\mathrm{VO}}_{2 \text { peak, }}$ $\left.\dot{\mathrm{VO}_{2}}: 1.4 \pm 0.1 \mathrm{~L} \cdot \mathrm{min}^{-1} ; p>0.05\right)$, with no differences reported within or between groups, or compared to set exercise intensity $(p>0.05)$. No significant differences were reported at baseline for all variables $(p>0.05)$. A significant interaction effect was found for mean $\operatorname{RER}\left(\mathrm{F}=4.66, p=0.003, \eta_{\mathrm{p}}{ }^{2}=0.28\right)$, with post-hoc analyses revealing a reduction in RER by week 8 for dGTE+ compared with week $4(p=0.004)$. When normalised data were considered, a significant overall between-group effect was found for RER $(\mathrm{F}=4.22, p=0.027$, $\left.\eta_{\mathrm{p}}{ }^{2}=0.26\right)$, highlighting a difference between dGTE and dGTE+ by week $8(p=0.034)$. 
Table 4. Mean fat oxidation measures at baseline (week 0), week 4 and 8 between intervention groups.

\begin{tabular}{|c|c|c|c|c|c|c|c|c|c|}
\hline \multirow[b]{2}{*}{ Variable } & \multicolumn{3}{|c|}{ PL } & \multicolumn{3}{|c|}{ dGTE } & \multicolumn{3}{|c|}{ dGTE+ } \\
\hline & Baseline & Week 4 & Week 8 & Baseline & Week 4 & Week 8 & Baseline & Week 4 & Week 8 \\
\hline $\mathrm{MFO}\left(\mathrm{mg} \cdot \mathrm{min}^{-1}\right)$ & $228.1 \pm 23.4$ & $219.5 \pm 34.1$ & $201.1 \pm 25.2$ & $199.0 \pm 30.8$ & $223.2 \pm 32.3$ & $224.4 \pm 27.8$ & $154.4 \pm 20.6$ & $176.6 \pm 19.3$ & $224.6 \pm 23.2 *$ \\
\hline $\mathrm{MFO} \cdot \mathrm{FFM}^{-1}\left(\mathrm{mg} \cdot \mathrm{min}^{-1}\right)$ & $4.7 \pm 0.7$ & $4.1 \pm 0.6$ & $3.7 \pm 0.5$ & $4.0 \pm 0.6$ & $4.4 \pm 0.6$ & $4.5 \pm 0.5$ & $3.2 \pm 0.4$ & $3.7 \pm 0.4$ & $4.7 \pm 0.5^{*}$ \\
\hline $\mathrm{FAT}_{\text {MAX }}\left(\% \dot{\mathrm{VO}}_{2 \text { peak }}\right)$ & $35.2 \pm 2.0$ & $38.6 \pm 2.2$ & $38.0 \pm 1.9$ & $36.5 \pm 6.0$ & $34.1 \pm 1.8$ & $36.5 \pm 2.9$ & $39.5 \pm 2.6$ & $34.4 \pm 1.3$ & $42.7 \pm 2.3$ \\
\hline $\mathrm{HR}$ at $\mathrm{FAT}_{\mathrm{MAX}}\left(\% \mathrm{HR}_{\max }\right)$ & $57.6 \pm 1.9$ & $58.5 \pm 2.1$ & $57.7 \pm 1.7$ & $56.3 \pm 1.5$ & $57.9 \pm 1.6$ & $56.8 \pm 1.7$ & $57.1 \pm 1.7$ & $57.5 \pm 2.1$ & $58.1 \pm 1.6$ \\
\hline $\mathrm{FAT}_{\mathrm{MIN}}\left(\% \mathrm{VO}_{2 \text { peak }}\right)$ & $61.7 \pm 4.3$ & $64.7 \pm 4.2$ & $56.1 \pm 4.0$ & $60.1 \pm 4.1$ & $54.9 \pm 3.4$ & $57.2 \pm 3.6$ & $58.3 \pm 2.6$ & $55.1 \pm 1.9$ & $67.6 \pm 3.6^{\#}$ \\
\hline
\end{tabular}

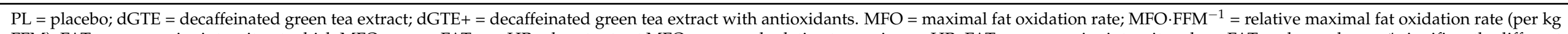

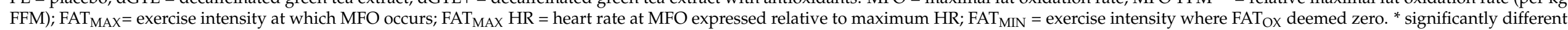
from baseline within group $(p \leq 0.024)$; \# significantly different to week 4 within group $(p=0.003)$.

Table 5. Mean steady state exercise measures at baseline (week 0), week 4 and 8 between intervention groups.

\begin{tabular}{|c|c|c|c|c|c|c|c|c|c|}
\hline \multirow[b]{2}{*}{ Variable } & \multicolumn{3}{|c|}{ PL } & \multicolumn{3}{|c|}{ dGTE } & \multicolumn{3}{|c|}{ dGTE+ } \\
\hline & Baseline & Week 4 & Week 8 & Baseline & Week 4 & Week 8 & Baseline & Week 4 & Week 8 \\
\hline RER & $0.91 \pm 0.01$ & $0.90 \pm 0.02$ & $0.92 \pm 0.02$ & $0.90 \pm 0.02$ & $0.92 \pm 0.02$ & $0.93 \pm 0.01$ & $0.92 \pm 0.01$ & $0.94 \pm 0.01$ & $0.89 \pm 0.01$ * \\
\hline $\mathrm{FAT}_{\mathrm{OX}}\left(\mathrm{mg} \cdot \mathrm{min}^{-1}\right)$ & $224.4 \pm 27.7$ & $237.3 \pm 48.9$ & $181.6 \pm 36.8$ & $222.3 \pm 41.4$ & $185.7 \pm 41.1$ & $161.3 \pm 31.1$ & $169.1 \pm 37.6$ & $152.6 \pm 45.8$ & $217.2 \pm 31.7$ \\
\hline $\mathrm{FAT}_{\mathrm{OX}} \cdot \mathrm{FFM}^{-1}\left(\mathrm{mg} \cdot \mathrm{min}^{-1}\right)$ & $4.1 \pm 0.5$ & $4.4 \pm 0.9$ & $3.3 \pm 0.6$ & $4.5 \pm 0.8$ & $3.6 \pm 0.7$ & $3.2 \pm 0.6$ & $3.4 \pm 0.7$ & $2.9 \pm 0.7$ & $4.5 \pm 0.7^{*}$ \\
\hline $\mathrm{CHO}_{\mathrm{OX}} \mathrm{ST}(\%)$ & $62.6 \pm 5.5$ & $61.2 \pm 5.2$ & $68.2 \pm 4.1$ & $64.4 \pm 3.9$ & $67.6 \pm 4.8$ & $63.5 \pm 5.3$ & $72.0 \pm 2.8$ & $71.7 \pm 2.1$ & $63.1 \pm 4.2$ \\
\hline $\mathrm{FAT}_{\mathrm{OX}} \mathrm{ST}(\%)$ & $31.5 \pm 5.0$ & $35.4 \pm 7.5$ & $28.3 \pm 6.0$ & $34.4 \pm 7.4$ & $32.2 \pm 5.7$ & $26.5 \pm 5.5$ & $27.8 \pm 5.5$ & $22.5 \pm 4.6$ & $36.8 \pm 5.3$ \\
\hline Total EE $\left(\mathrm{kcal} . \mathrm{hr}^{-1}\right)$ & $446.6 \pm 27.6$ & $420.4 \pm 25.8$ & $435.8 \pm 27.1$ & $421.0 \pm 30.4$ & $407.5 \pm 31.8$ & $404.4 \pm 29.0$ & $377.9 \pm 28.9$ & $383.3 \pm 39.4$ & $374.4 \pm 30.3$ \\
\hline CHOcont. EE (\%) & $69.9 \pm 4.0$ & $67.1 \pm 6.9$ & $75.2 \pm 5.2$ & $69.1 \pm 6.0$ & $74.3 \pm 5.8$ & $77.4 \pm 4.6$ & $75.0 \pm 4.6$ & $79.7 \pm 4.5$ & $65.5 \pm 4.6^{*}$ \\
\hline FATcont. EE (\%) & $30.3 \pm 4.1$ & $33.4 \pm 6.8$ & $25.3 \pm 5.1$ & $31.4 \pm 5.8$ & $26.5 \pm 5.4$ & $23.3 \pm 4.4$ & $25.0 \pm 4.6$ & $21.0 \pm 4.1$ & $34.6 \pm 4.7$ * \\
\hline
\end{tabular}

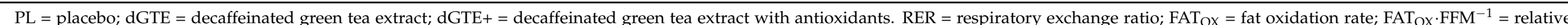

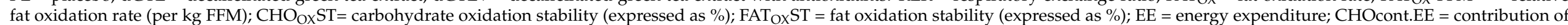
carbohydrate to total EE; FATcont.EE = contribution of fat to total EE. * significantly different to week 4 within condition $(p \leq 0.036)$. 

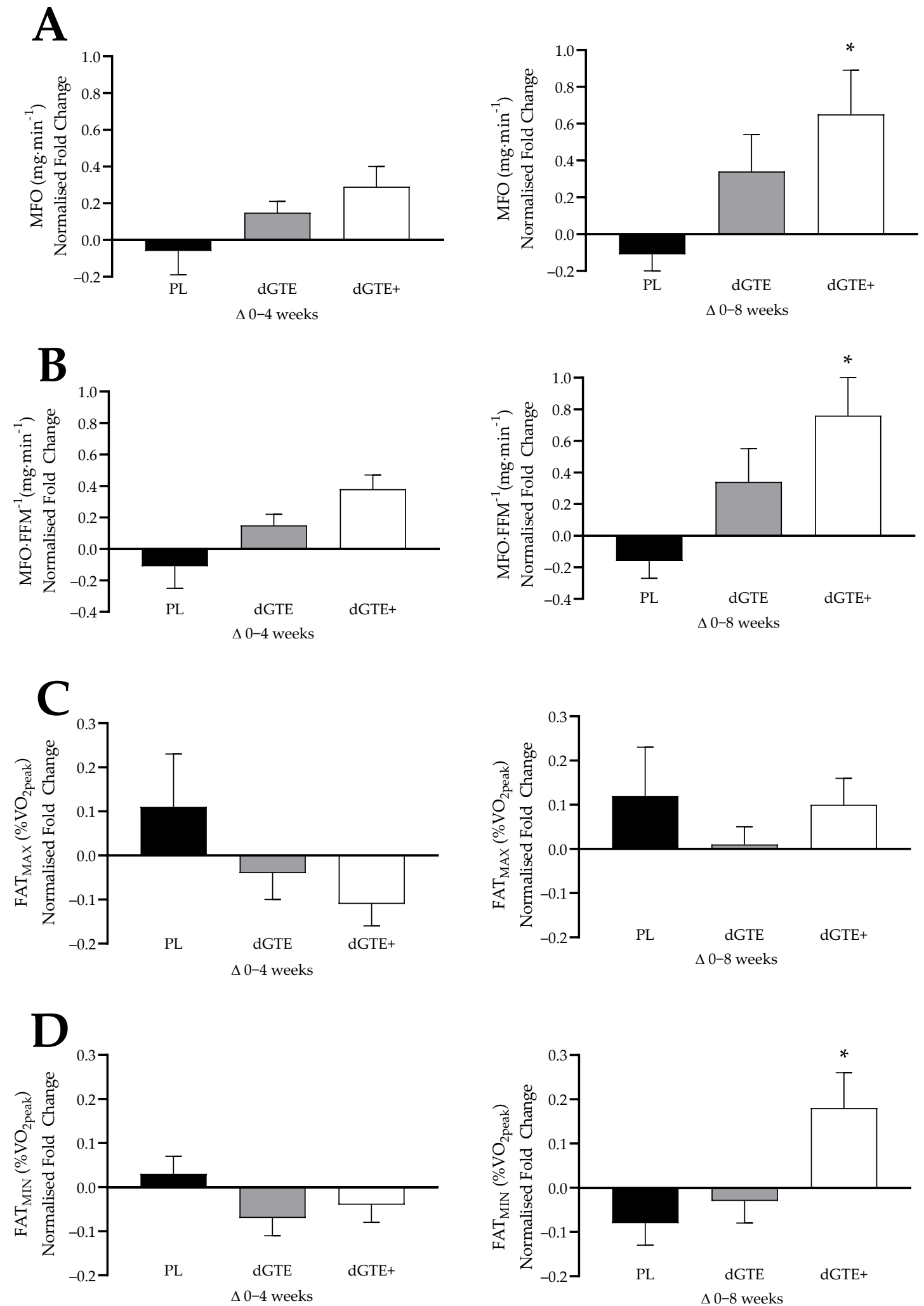

Figure 1. Mean normalised fold change for FAT $\mathrm{MAX}_{\mathrm{X}}$ test parameters between groups by week 4 ( $\Delta 0-4$ weeks) and week 8 ( $\triangle 0-8$ weeks) of the intervention; showing (A) maximal fat oxidation rate (MFO), (B) relative maximal fat oxidation rate per $\mathrm{kg}$ FFM (MFO·FFM $\left.{ }^{-1}\right),($ C) exercise intensity at which MFO occurs (FAT $\mathrm{MAX}$ ), and (D) exercise intensity where fat oxidation deemed zero $\left(\mathrm{FAT}_{\mathrm{MIN}}\right) . \mathrm{PL}=$ placebo; $\mathrm{dGTE}=$ decaffeinated green tea extract; $\mathrm{dGTE}+=$ decaffeinated green tea extract with antioxidants. ${ }^{*}$ significantly different from PL $(p \leq 0.034)$. 

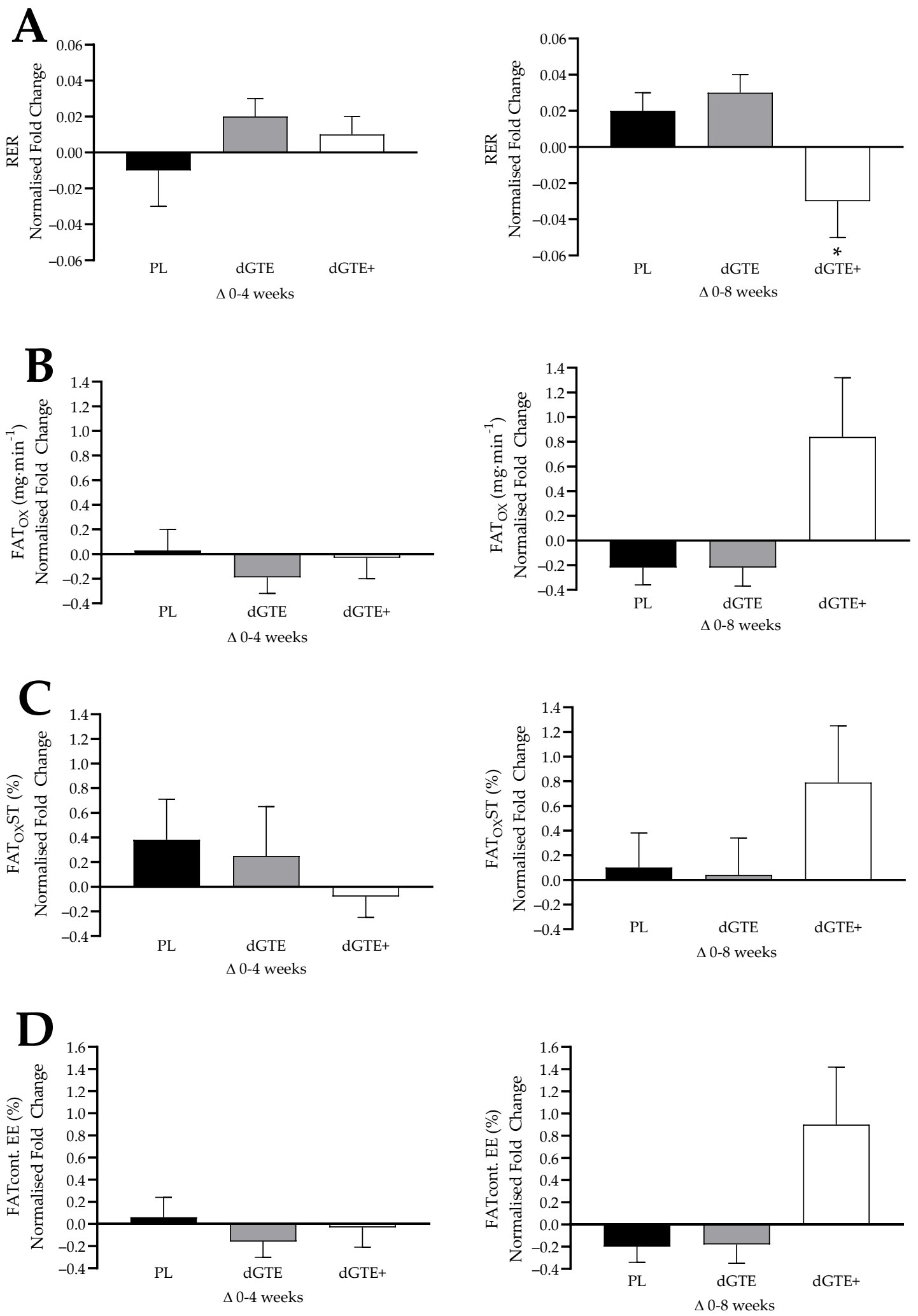

Figure 2. Mean normalised fold change for fat oxidation parameters during steady state exercise between groups by week 4 $(\Delta 0-4$ weeks) and week 8 ( $\Delta 0-8$ weeks) of the intervention; showing (A) respiratory exchange ratio (RER), (B) fat oxidation rate $\left(\mathrm{FAT}_{\mathrm{OX}}\right),(\mathrm{C})$ fat oxidation stability $\left(\mathrm{FAT}_{\mathrm{OX}} \mathrm{ST}\right)$, and $(\mathrm{D})$ contribution of fat to total energy expenditure (FATcont.EE). PL = placebo; $\mathrm{dGTE}=$ decaffeinated green tea extract; $\mathrm{dGTE}+=$ decaffeinated green tea extract with antioxidants. ${ }^{*}$ significantly different to dGTE only $(p=0.034)$. 
In terms of normalised fold change (Figure 1), a significant effect for MFO was reported overall by week $8\left(\mathrm{~F}=3.81, p=0.037, \eta_{\mathrm{p}}{ }^{2}=0.24\right)$, but not by week $4(p=0.113)$. Normalised MFO was significantly greater with $\mathrm{dGTE}+$ only $(+0.65 \pm 0.25$ fold change $)$ compared with PL $(-0.11 \pm 0.09$ fold change; $p=0.034)$ across the intervention. Similarly, normalised relative MFO was significantly different between groups by week $8(\mathrm{~F}=4.16, p=0.028$, $\left.\eta_{\mathrm{p}}{ }^{2}=0.26\right)$ but not by week $4(p=0.073)$, again with a highlighted increase for dGTE+ $(+0.76 \pm 0.24$ fold change $)$ compared with PL $(-0.16 \pm 0.11$ fold change; $p=0.026)$.

Whilst a significant interaction effect was also found for mean FAT $_{\text {Ox }}$ during steady state exercise $\left(\mathrm{F}=3.15, p=0.022, \eta_{\mathrm{p}}{ }^{2}=0.21\right)$, no significant post-hoc analyses were found, despite $\mathrm{a}+28.4 \%$ increase in steady state FAT $_{\mathrm{Ox}}$ by week 8 with $\mathrm{dGTE}+(p>0.05)$. However, a significant interaction was found with relative FAT $_{\mathrm{OX}}\left(\mathrm{F}=3.98, p=0.007, \eta_{\mathrm{p}}{ }^{2}=0.25\right)$, with $\mathrm{a}+54.2 \%$ increase in $\mathrm{FAT}_{\mathrm{OX}} \cdot \mathrm{FFM}^{-1}$ by week 8 compared to week 4 for dGTE+ only $(p=0.036)$. Normalised data also highlighted a significant between-group effect for FAT OX by week $8\left(\mathrm{~F}=4.050, p=0.031, \eta_{\mathrm{p}}^{2}=0.252\right)$ with a $+0.84 \pm 0.48$ fold change for dGTE + compared with both PL $(-0.22 \pm 0.14)$ and dGTE $(-0.22 \pm 0.15)$. However, post-hoc analyses only revealed a trend between PL and dGTE $(p=0.064)$ and dGTE and dGTE+ $(p=0.063)$. A similar pattern was found for normalised $\mathrm{FAT}_{\mathrm{OX}} \cdot \mathrm{FFM}^{-1}(\mathrm{~F}=3.93, p=0.033$, $\eta_{\mathrm{p}}{ }^{2}=0.25$ ), with no significant post-hoc analyses (PL compared with dGTE, $p=0.071$; dGTE compared with dGTE+, $p=0.068$ ).

No significant effects were found for mean carbohydrate oxidation stability, despite a noted $-11.9 \%$ reduction by week 8 for dGTE+ $(p>0.05)$. Similarly, mean fat oxidation stability did not significantly improve with either dGTE formula, despite an observed trend (interaction effect: $\mathrm{F}=2.36, p=0.066, \eta_{\mathrm{p}}{ }^{2}=0.16$ ) by week 8 for $\mathrm{dGTE}+$. No significant differences were observed for normalised data for carbohydrate or fat oxidation stability at week 4 or $8(p>0.05)$. No significant differences were reported for EE within or between groups $(p>0.05)$. However, a significant interaction effect was found for both the contribution of carbohydrate $\left(\mathrm{F}=4.37, p=0.004, \eta_{\mathrm{p}}{ }^{2}=0.27\right)$ and the contribution of fat $\left(\mathrm{F}=4.29, p=0.005, \eta_{\mathrm{p}}{ }^{2}=0.26\right)$ to $\mathrm{EE}$, highlighting a $-17.8 \%$ reduction in carbohydrate contribution $(p=0.008)$ and $\mathrm{a}+64.8 \%$ increase in fat contribution $(p=0.006)$ to EE for dGTE at week 8 compared to week 4 , respectively. When normalised data were considered, a significant between-group effect was observed for both carbohydrate $(\mathrm{F}=3.66, p=0.041$, $\left.\eta_{\mathrm{p}}{ }^{2}=0.23\right)$ and fat $\left(\mathrm{F}=3.73, p=0.039, \eta_{\mathrm{p}}{ }^{2}=0.237\right)$ contribution to EE by week 8 . However, post-hoc analyses only indicated a difference between dGTE and dGTE+ for carbohydrate contribution to $\mathrm{EE}(p=0.043)$, but not fat contribution $(p=0.083)$.

\subsection{Body Composition, HR and Blood Pressure}

Mean body composition, resting $\mathrm{HR}$ and blood pressure measures are shown in Table 6. No significant differences were reported at baseline for all variables $(p>0.05)$. No significant differences were observed for body mass, BMI, body fat, fat-free mass, fat mass, WHR, SAD, VFA or blood pressure within or between groups $(p>0.05)$. A main effect was reported for waist circumference $\left(\mathrm{F}=4.65, p=0.014, \eta_{\mathrm{p}}{ }^{2}=0.16\right)$, with a $-0.8 \pm 0.2 \mathrm{~cm}$ relative decrease at week 4 compared to baseline for PL only $(p=0.019)$ A main effect was also reported for abdominal circumference $\left(F=6.19, p=0.004, \eta_{p}{ }^{2}=0.21\right)$, with a $-1.6 \pm 0.4 \mathrm{~cm}$ relative decrease by week 4 for dGTE compared to baseline $(p=0.004)$. Resting HR was significantly reduced by week 4 for dGTE+ compared with dGTE only (main interaction; $\mathrm{F}=2.61, p=0.047, \eta_{\mathrm{p}}{ }^{2}=0.18$ ). 
Table 6. Body composition, resting heart rate and blood pressure measures at baseline (week 0), week 4 and 8 between intervention groups.

\begin{tabular}{|c|c|c|c|c|c|c|c|c|c|}
\hline & \multicolumn{3}{|c|}{ PL } & \multicolumn{3}{|c|}{ dGTE } & \multicolumn{3}{|c|}{ dGTE+ } \\
\hline Variable & Baseline & Week 4 & Week 8 & Baseline & Week 4 & Week 8 & Baseline & Week 4 & Week 8 \\
\hline $\begin{array}{l}\text { Body mass } \\
(\mathrm{kg})\end{array}$ & $82.7 \pm 4.3$ & $82.5 \pm 4.3$ & $82.6 \pm 4.2$ & $74.3 \pm 2.5$ & $73.8 \pm 2.3$ & $73.9 \pm 2.4$ & $75.6 \pm 3.1$ & $75.3 \pm 3.2$ & $75.1 \pm 3.1$ \\
\hline $\begin{array}{l}\mathrm{BMI} \\
\left(\mathrm{kg} \cdot \mathrm{m}^{2}\right)\end{array}$ & $28.2 \pm 1.4$ & $28.1 \pm 1.4$ & $28.2 \pm 1.3$ & $26.8 \pm 0.8$ & $26.7 \pm 0.9$ & $26.7 \pm 0.9$ & $27.1 \pm 0.8$ & $27.1 \pm 0.8$ & $27.0 \pm 0.9$ \\
\hline $\begin{array}{l}\text { Body fat } \\
(\%)\end{array}$ & $32.0 \pm 2.6$ & $31.7 \pm 2.5$ & $32.2 \pm 2.7$ & $33.2 \pm 2.2$ & $32.7 \pm 2.3$ & $32.9 \pm 2.2$ & $36.3 \pm 2.1$ & $36.1 \pm 2.1$ & $35.8 \pm 2.2$ \\
\hline FFM (kg) & $56.1 \pm 3.4$ & $55.9 \pm 3.2$ & $55.5 \pm 3.1$ & $49.6 \pm 2.5$ & $49.7 \pm 2.5$ & $49.5 \pm 2.4$ & $48.0 \pm 2.5$ & $48.0 \pm 2.5$ & $48.2 \pm 2.4$ \\
\hline FM (kg) & $26.6 \pm 2.8$ & $26.3 \pm 2.7$ & $26.7 \pm 2.8$ & $24.6 \pm 1.8$ & $24.1 \pm 1.9$ & $24.3 \pm 1.8$ & $27.3 \pm 2.1$ & $27.3 \pm 2.3$ & $27.0 \pm 2.4$ \\
\hline WHR & $0.84 \pm 0.03$ & $0.83 \pm 0.02$ & $0.83 \pm 0.03$ & $0.82 \pm 0.02$ & $0.82 \pm 0.02$ & $0.83 \pm 0.02$ & $0.80 \pm 0.01$ & $0.80 \pm 0.01$ & $0.80 \pm 0.01$ \\
\hline $\begin{array}{l}\text { Waist C } \\
(\mathrm{cm})\end{array}$ & $89.5 \pm 3.2$ & $88.7 \pm 3.1^{*}$ & $89.0 \pm 3.0$ & $85.7 \pm 2.4$ & $85.3 \pm 2.5$ & $85.8 \pm 2.6$ & $85.2 \pm 2.0$ & $84.9 \pm 1.9$ & $84.9 \pm 2.0$ \\
\hline Ab. C (cm) & $102.0 \pm 2.3$ & $101.8 \pm 2.6$ & $101.9 \pm 2.6$ & $100.8 \pm 2.8$ & $99.2 \pm 2.7^{*}$ & $99.7 \pm 2.8$ & $100.9 \pm 2.9$ & $100.4 \pm 2.9$ & $100.1 \pm 2.9$ \\
\hline $\mathrm{SAD}(\mathrm{cm})$ & $23.3 \pm 0.9$ & $23.1 \pm 0.9$ & $23.0 \pm 0.9$ & $21.6 \pm 0.5$ & $21.1 \pm 0.7$ & $21.3 \pm 0.7$ & $21.9 \pm 0.7$ & $21.6 \pm 0.7$ & $21.3 \pm 0.8$ \\
\hline VFA $\left(\mathrm{cm}^{2}\right)$ & $\begin{array}{c}1740.1 \pm \\
140.8\end{array}$ & $\begin{array}{c}1739.3 \pm \\
151.8\end{array}$ & $\begin{array}{c}1714.8 \pm \\
135.7\end{array}$ & $\begin{array}{c}1576.0 \pm \\
116.1\end{array}$ & $\begin{array}{c}1554.7 \pm \\
134.4\end{array}$ & $\begin{array}{c}1550.1 \pm \\
151.1\end{array}$ & $\begin{array}{c}1778.4 \pm \\
143.8\end{array}$ & $\begin{array}{c}1748.3 \pm \\
136.6\end{array}$ & $\begin{array}{c}1699.6 \pm \\
161.7\end{array}$ \\
\hline $\begin{array}{l}\text { HR } \\
\left(b \cdot \min ^{-1}\right)\end{array}$ & $68 \pm 3$ & $65 \pm 2$ & $69 \pm 4$ & $62 \pm 3$ & $68 \pm 4$ & $65 \pm 2$ & $63 \pm 2$ & $58 \pm 1 \#$ & $60 \pm 2$ \\
\hline $\begin{array}{l}\text { SBP } \\
(\mathrm{mmHg})\end{array}$ & $130 \pm 9$ & $128 \pm 9$ & $128 \pm 8$ & $117 \pm 3$ & $120 \pm 4$ & $115 \pm 4$ & $114 \pm 6$ & $113 \pm 4$ & $111 \pm 4$ \\
\hline $\begin{array}{l}\text { DBP } \\
(\mathrm{mmHg})\end{array}$ & $84 \pm 5$ & $84 \pm 6$ & $84 \pm 5$ & $78 \pm 2$ & $78 \pm 1$ & $78 \pm 2$ & $77 \pm 3$ & $77 \pm 3$ & $75 \pm 2$ \\
\hline
\end{tabular}

$\mathrm{PL}=$ placebo; $\mathrm{dGTE}=$ decaffeinated green tea extract; $\mathrm{dGTE}+=$ decaffeinated green tea extract with antioxidants. $\mathrm{BMI}=$ body mass index; $\mathrm{FFM}=$ fat free mass; $\mathrm{FM}=$ fat mass; $\mathrm{WHR}=$ waist to hip ratio; waist $\mathrm{C}=$ waist circumference; $\mathrm{Ab}$. $\mathrm{C}=$ abdominal circumference; $\mathrm{SAD}=$ sagittal abdominal diameter; VFA = estimated visceral fat area; $\mathrm{HR}=$ resting heart rate; $\mathrm{SBP}=$ systolic blood pressure; $\mathrm{DBP}=$ diastolic blood pressure. * significantly different to baseline within group only $(p \leq 0.019)$. \# significantly different to dGTE at timepoint $(p=0.05)$.

\subsection{Blood Analytes}

Mean serum cardio-metabolic markers, including normalised fold change, are shown in Tables 7 and 8. No significant differences were reported at baseline for all variables $(p>0.05)$, except adiponectin. Mean adiponectin levels for dGTE+ were significantly greater compared to PL at baseline $(p=0.036)$, week $4(p=0.021)$ and week $8(p=0.049)$. No other main effects were found for all variables, except for LDL-c, where a main interaction effect was observed $\left(F=2.75, p=0.039, \eta_{p}{ }^{2}=0.19\right)$. However, post-hoc analyses did not reveal any significant differences for LDL-c within or between groups despite a relative $-0.38 \pm 0.22 \mathrm{mmol} \cdot \mathrm{L}^{-1}$ reduction for dGTE + , and a relative $+0.30 \pm 0.12 \mathrm{mmol} \cdot \mathrm{L}^{-1}$ increase for dGTE over the intervention. When data were normalised, a significant interaction effect was found for LDL-c over the intervention $\left(F=3.95, p=0.033, \eta_{p}{ }^{2}=0.25\right)$ highlighting the difference between dGTE and dGTE+ only $(p=0.038)$.

Mean serum liver function markers, including normalised fold change, are shown in Tables 9 and 10. All mean data were considered within normal reference ranges for all variables. No significant effects were found for ALT, AST or bilirubin, despite relative reductions of $-16.22 \pm 11.52 \mathrm{U} \cdot \mathrm{L}^{-1},-8.22 \pm 7.43 \mathrm{U} \cdot \mathrm{L}^{-1}$ and $-0.89 \pm 1.78 \mu \mathrm{mol} \cdot \mathrm{L}^{-1}$, respectively, by week 8 for PL $(p>0.05)$. However, ALP significantly reduced by $-6.67 \pm 3.03 \mathrm{U} \cdot \mathrm{L}^{-1}$ over time $\left(\mathrm{F}=6.63, p=0.003, \eta_{\mathrm{p}}{ }^{2}=0.22\right)$ for PL only $(p \leq 0.025$ for both baseline and week 4 compared with week 8 ). When data were normalised, a significant between-group effect was reported overall for ALT only $\left(\mathrm{F}=4.88, p=0.017, \eta_{\mathrm{p}}{ }^{2}=0.29\right)$, with the highlighted difference between PL and dGTE+ $(p=0.015)$ likely explained by the relative decrease for ALT in PL over time. All liver enzymes, and bilirubin, were unaffected by both dGTE and dGTE+ supplementation. 
Table 7. Mean serum cardio-metabolic markers at baseline (week 0), week 4 and 8 between intervention groups.

\begin{tabular}{|c|c|c|c|c|c|c|c|c|c|}
\hline \multirow[b]{2}{*}{ Variable } & \multicolumn{3}{|c|}{ PL } & \multicolumn{3}{|c|}{ dGTE } & \multicolumn{3}{|c|}{ dGTE+ } \\
\hline & Baseline & Week 4 & Week 8 & Baseline & Week 4 & Week 8 & Baseline & Week 4 & Week 8 \\
\hline Glucose $\left(\mathrm{mmol} \cdot \mathrm{L}^{-1}\right)$ & $5.36 \pm 0.10$ & $5.37 \pm 0.14$ & $5.41 \pm 0.13$ & $5.78 \pm 0.17$ & $5.53 \pm 0.21$ & $5.57 \pm 0.22$ & $5.32 \pm 0.16$ & $5.29 \pm 0.13$ & $5.23 \pm 0.15$ \\
\hline Insulin $\left(\mathrm{pmol} \cdot \mathrm{L}^{-1}\right)$ & $61.67 \pm 12.34$ & $66.33 \pm 13.66$ & $71.33 \pm 15.38$ & $48.44 \pm 6.04$ & $48.22 \pm 5.91$ & $43.78 \pm 4.85$ & $39.67 \pm 5.47$ & $49.00 \pm 7.00$ & $43.22 \pm 7.01$ \\
\hline$\% \mathrm{~S}$ & $114.63 \pm 23.30$ & $100.87 \pm 15.19$ & $91.07 \pm 11.66$ & $121.32 \pm 15.36$ & $121.12 \pm 14.40$ & $132.39 \pm 19.49$ & $155.48 \pm 21.10$ & $131.66 \pm 25.71$ & $150.53 \pm 26.62$ \\
\hline HOMA-IR & $1.19 \pm 0.23$ & $1.26 \pm 0.26$ & $1.35 \pm 0.29$ & $0.94 \pm 0.12$ & $0.93 \pm 0.12$ & $0.85 \pm 0.09$ & $0.76 \pm 0.11$ & $0.95 \pm 0.12$ & $0.84 \pm 0.13$ \\
\hline Leptin $\left(\mathrm{ng} \cdot \mathrm{mL}^{-1}\right)$ & $15.88 \pm 3.83$ & $16.69 \pm 4.00$ & $18.78 \pm 3.61$ & $17.59 \pm 4.28$ & $18.58 \pm 5.39$ & $17.46 \pm 3.75$ & $24.66 \pm 5.50$ & $24.19 \pm 6.25$ & $25.21 \pm 5.89$ \\
\hline Adiponectin $\left(\mu \mathrm{g} \cdot \mathrm{mL}^{-1}\right)$ & $5.94 \pm 1.50$ & $5.79 \pm 1.47$ & $5.82 \pm 1.35$ & $9.06 \pm 1.16$ & $8.90 \pm 1.30$ & $9.39 \pm 1.45$ & $11.17 \pm 1.40 *$ & $11.54 \pm 1.37 *$ & $11.08 \pm 1.50 *$ \\
\hline Glycerol $\left(\mu \mathrm{mol} \cdot \mathrm{L}^{-1}\right)$ & $25.44 \pm 3.09$ & $27.56 \pm 3.50$ & $27.00 \pm 2.40$ & $28.00 \pm 2.70$ & $36.44 \pm 4.61$ & $29.89 \pm 3.89$ & $31.56 \pm 6.31$ & $29.78 \pm 3.42$ & $31.11 \pm 3.58$ \\
\hline $\mathrm{Chol}_{\mathrm{TOT}}\left(\mathrm{mmol} \cdot \mathrm{L}^{-1}\right)$ & $5.06 \pm 0.27$ & $5.04 \pm 0.21$ & $5.17 \pm 0.20$ & $5.16 \pm 0.25$ & $5.34 \pm 0.28$ & $5.53 \pm 0.33$ & $5.31 \pm 0.30$ & $5.28 \pm 0.25$ & $5.22 \pm 0.21$ \\
\hline $\mathrm{TG}\left(\mathrm{mmol} \cdot \mathrm{L}^{-1}\right)$ & $1.11 \pm 0.22$ & $1.11 \pm 0.22$ & $1.34 \pm 0.30$ & $1.03 \pm 0.22$ & $1.03 \pm 0.19$ & $1.02 \pm 0.19$ & $0.92 \pm 0.13$ & $0.74 \pm 0.11$ & $0.89 \pm 0.13$ \\
\hline HDL-c $\left(\mathrm{mmol} \cdot \mathrm{L}^{-1}\right)$ & $1.40 \pm 0.16$ & $1.37 \pm 0.14$ & $1.33 \pm 0.13$ & $1.65 \pm 0.16$ & $1.68 \pm 0.17$ & $1.73 \pm 0.19$ & $1.75 \pm 0.15$ & $1.82 \pm 0.18$ & $1.76 \pm 0.14$ \\
\hline $\mathrm{LDL}-\mathrm{c}\left(\mathrm{mmol} \cdot \mathrm{L}^{-1}\right)$ & $3.13 \pm 0.26$ & $3.17 \pm 0.20$ & $3.20 \pm 0.16$ & $3.04 \pm 0.19$ & $3.19 \pm 0.19$ & $3.34 \pm 0.22$ & $3.42 \pm 0.28$ & $3.12 \pm 0.26$ & $3.04 \pm 0.25$ \\
\hline
\end{tabular}

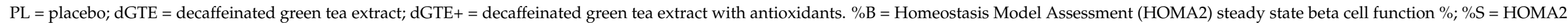

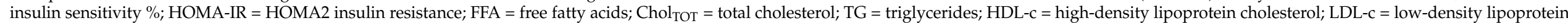
cholesterol. * significantly different to PL at timepoint $(p \leq 0.049)$.

Table 8. Normalised mean fold change in serum cardio-metabolic markers by week $4(\mathrm{~N} \Delta 0-4)$, and week $8(\mathrm{~N} \Delta 0-8)$.

\begin{tabular}{|c|c|c|c|c|c|c|}
\hline & & $\mathrm{N} \Delta 0-4$ & & & $\mathrm{~N} \Delta 0-8$ & \\
\hline Variable & PL & dGTE & dGTE+ & PL & dGTE & dGTE+ \\
\hline Glucose $\left(\mathrm{mmol} \cdot \mathrm{L}^{-1}\right)$ & $+0.00 \pm 0.02$ & $-0.04 \pm 0.03$ & $+0.00 \pm 0.02$ & $+0.01 \pm 0.02$ & $-0.04 \pm 0.02$ & $-0.02 \pm 0.02$ \\
\hline Insulin $\left(\mathrm{pmol} \cdot \mathrm{L}^{-1}\right)$ & $+0.28 \pm 0.24$ & $+0.03 \pm 0.08$ & $+0.22 \pm 0.12$ & $+0.45 \pm 0.34$ & $-0.04 \pm 0.12$ & $+0.06 \pm 0.10$ \\
\hline$\% \mathrm{~B}$ & $+0.08 \pm 0.09$ & $+0.12 \pm 0.08$ & $+0.18 \pm 0.08$ & $+0.13 \pm 0.12$ & $+0.05 \pm 0.07$ & $+0.11 \pm 0.07$ \\
\hline$\% \mathrm{~S}$ & $+0.01 \pm 0.15$ & $+0.03 \pm 0.08$ & $-0.16 \pm 0.06$ & $-0.07 \pm 0.13$ & $+0.16 \pm 0.15$ & $-0.05 \pm 0.07$ \\
\hline HOMA-IR & $+0.17 \pm 0.16$ & $+0.02 \pm 0.08$ & $+0.26 \pm 0.10$ & $+0.29 \pm 0.21$ & $-0.02 \pm 0.12$ & $+0.09 \pm 0.08$ \\
\hline Leptin (ng. $\mathrm{mL}^{-1}$ ) & $+0.13 \pm 0.20$ & $-0.02 \pm 0.07$ & $+0.00 \pm 0.09$ & $+0.26 \pm 0.13$ & $+0.02 \pm 0.08$ & $+0.02 \pm 0.11$ \\
\hline Adiponectin $\left(\mu \mathrm{g} \cdot \mathrm{mL}^{-1}\right)$ & $-0.02 \pm 0.01$ & $-0.03 \pm 0.05$ & $+0.05 \pm 0.04$ & $+0.01 \pm 0.05$ & $+0.02 \pm 0.04$ & $-0.02 \pm 0.04$ \\
\hline Glycerol $\left(\mu \mathrm{mol} \cdot \mathrm{L}^{-1}\right)$ & $+0.15 \pm 0.15$ & $+0.33 \pm 0.16$ & $+0.20 \pm 0.24$ & $+0.11 \pm 0.09$ & $+0.11 \pm 0.14$ & $+0.22 \pm 0.23$ \\
\hline $\mathrm{Chol}_{\mathrm{TOT}}\left(\mathrm{mmol} \cdot \mathrm{L}^{-1}\right)$ & $+0.00 \pm 0.02$ & $+0.04 \pm 0.03$ & $+0.00 \pm 0.03$ & $+0.03 \pm 0.03$ & $+0.07 \pm 0.03$ & $-0.01 \pm 0.02$ \\
\hline $\mathrm{TG}\left(\mathrm{mmol} \cdot \mathrm{L}^{-1}\right)$ & $+0.03 \pm 0.11$ & $+0.05 \pm 0.11$ & $-0.15 \pm 0.11$ & $+0.19 \pm 0.16$ & $+0.02 \pm 0.09$ & $-0.03 \pm 0.06$ \\
\hline HDL-c $\left(\mathrm{mmol} \cdot \mathrm{L}^{-1}\right)$ & $-0.01 \pm 0.04$ & $+0.02 \pm 0.03$ & $+0.04 \pm 0.04$ & $-0.03 \pm 0.04$ & $+0.04 \pm 0.03$ & $+0.01 \pm 0.04$ \\
\hline LDL-c $\left(\mathrm{mmol} \cdot \mathrm{L}^{-1}\right)$ & $+0.03 \pm 0.04$ & $+0.05 \pm 0.03$ & $-0.07 \pm 0.06$ & $+0.05 \pm 0.06$ & $+0.10 \pm 0.04$ & $-0.09 \pm 0.06^{*}$ \\
\hline
\end{tabular}

$\mathrm{PL}=$ placebo; $\mathrm{dGTE}=$ decaffeinated green tea extract; $\mathrm{dGTE}+=$ decaffeinated green tea extract with antioxidants. ${ }^{*}$ denotes significantly different to dGTE $(p=0.038)$. 
Table 9. Mean serum liver function markers at baseline (week 0), week 4 and 8 between intervention groups.

\begin{tabular}{|c|c|c|c|c|c|c|c|c|c|}
\hline \multirow[b]{2}{*}{ Variable } & \multicolumn{3}{|c|}{ PL } & \multicolumn{3}{|c|}{ dGTE } & \multicolumn{3}{|c|}{ dGTE+ } \\
\hline & Baseline & Week 4 & Week 8 & Baseline & Week 4 & Week 8 & Baseline & Week 4 & Week 8 \\
\hline $\operatorname{ALT}\left(\mathrm{U} \cdot \mathrm{L}^{-1}\right)$ & $56.89 \pm 23.14$ & $47.22 \pm 15.86$ & $40.67 \pm 11.72$ & $34.56 \pm 2.69$ & $28.89 \pm 1.62$ & $34.22 \pm 2.41$ & $28.33 \pm 1.41$ & $30.78 \pm 1.82$ & $31.33 \pm 1.85$ \\
\hline $\operatorname{AST}\left(\mathrm{U} \cdot \mathrm{L}^{-1}\right)$ & $28.33 \pm 10.70$ & $37.11 \pm 15.58$ & $20.11 \pm 3.51$ & $19.78 \pm 1.23$ & $17.11 \pm 1.62$ & $21.11 \pm 1.35$ & $19.11 \pm 2.12$ & $18.22 \pm 1.42$ & $19.33 \pm 1.27$ \\
\hline $\operatorname{ALP}\left(\mathrm{U} \cdot \mathrm{L}^{-1}\right)$ & $66.89 \pm 7.13 *$ & $65.22 \pm 5.96^{*}$ & $60.22 \pm 6.15$ & $60.11 \pm 3.36$ & $60.00 \pm 3.76$ & $59.00 \pm 4.20$ & $59.44 \pm 3.70$ & $60.67 \pm 4.18$ & $56.67 \pm 3.82$ \\
\hline Bilirubin $\left(\mu \mathrm{mol} \cdot \mathrm{L}^{-1}\right)$ & $10.78 \pm 2.26$ & $9.33 \pm 1.80$ & $9.89 \pm 1.14$ & $9.67 \pm 0.94$ & $9.89 \pm 0.92$ & $10.00 \pm 1.04$ & $7.78 \pm 0.81$ & $7.56 \pm 0.87$ & $8.44 \pm 0.67$ \\
\hline
\end{tabular}

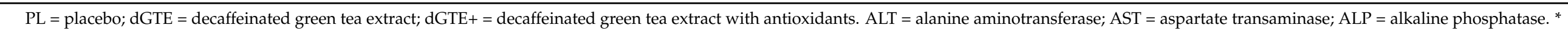
significantly different to week 8 within group $(p \leq 0.025)$.

Table 10. Normalised mean fold change in serum liver function markers by week $4(\mathrm{~N} \Delta 0-4)$, and week $8(\mathrm{~N} \Delta 0-8)$.

\begin{tabular}{|c|c|c|c|c|c|c|}
\hline & & $\mathrm{N} \Delta 0-4$ & & & $\mathrm{~N} \Delta 0-8$ & \\
\hline Variable & PL & dGTE & dGTE+ & PL & dGTE & dGTE+ \\
\hline $\operatorname{ALT}\left(\mathrm{U} \cdot \mathrm{L}^{-1}\right)$ & $-0.06 \pm 0.12$ & $-0.15 \pm 0.04$ & $+0.11 \pm 0.10$ & $-0.15 \pm 0.06$ & $+0.01 \pm 0.06$ & $+0.11 \pm 0.07$ * \\
\hline AST $\left(U \cdot L^{-1}\right)$ & $+0.49 \pm 0.62$ & $-0.12 \pm 0.09$ & $+0.02 \pm 0.11$ & $-0.09 \pm 0.10$ & $+0.08 \pm 0.05$ & $+0.10 \pm 0.12$ \\
\hline $\operatorname{ALP}\left(\mathrm{U} \cdot \mathrm{L}^{-1}\right)$ & $-0.01 \pm 0.02$ & $+0.00 \pm 0.03$ & $+0.02 \pm 0.02$ & $-0.09 \pm 0.04$ & $-0.02 \pm 0.03$ & $-0.05 \pm 0.02$ \\
\hline Bilirubin $\left(\mu \mathrm{mol} \cdot \mathrm{L}^{-1}\right)$ & $-0.02 \pm 0.14$ & $+0.07 \pm 0.13$ & $+0.02 \pm 0.11$ & $+0.08 \pm 0.15$ & $+0.09 \pm 0.15$ & $+0.14 \pm 0.09$ \\
\hline
\end{tabular}

$\mathrm{PL}=$ placebo; dGTE = decaffeinated green tea extract; dGTE $+=$ decaffeinated green tea extract with antioxidants. ${ }^{*}$ denotes significantly different to PL $(p=0.015)$. 


\section{Discussion}

This study aimed to assess the longer-term impact of moderate dGTE supplementation (with or without antioxidant nutrients) on fat oxidation, body composition and cardiometabolic risk factors in overweight, but healthy individuals engaged in regular exercise. The findings from the current study demonstrated that a moderate dose of dGTE consumed over 8 weeks did not result in a statistically significant change on fat oxidation rates, but did produce a non-significant increase in both MFO $(+12.7 \%)$ and relative MFO $(+13.9 \%)$. In contrast, the use of a novel dGTE + formula, containing antioxidants in addition to dGTE, had a significant impact (moderate-large effect size) on MFO (+45.5\%) and relative MFO $(+49.4 \%)$ by week 8 of supplementation in recreationally active, overweight individuals. Additionally, a large effect size for $\mathrm{FAT}_{\mathrm{MIN}}(+22.5 \%)$ was observed with dGTE+ over the last 4 weeks of this study. Metabolic changes were also observed by week 8 for dGTE+, with a large effect size reported for improvements in steady state substrate utilisation, evidenced by lower mean RER $(-5.3 \%)$, improved relative FAT $_{\mathrm{OX}}(+55.2 \%)$ and increased the contribution of fat to total EE $(+64.8 \%)$. Collectively, these findings suggest that a novel $\mathrm{dGTE}+$ formula positively influenced fat oxidation in overweight individuals, particularly in the final 4 weeks of this study.

With regards to the methodological approaches to $\mathrm{FAT}_{\mathrm{OX}}$ measures, concerns have been noted in the literature pertinent to reliability and variability [53-55]. This was also observed in the current study with dGTE + improving steady state $\mathrm{FAT}_{\mathrm{Ox}}$ by $+28.4 \%$, which was comparable to previous work undertaken [25], despite being non-significant. Whilst mean MFO and steady state $\mathrm{FAT}_{\mathrm{OX}}$ rates in the current study were comparable to previous research with untrained [56], active [57-59] or overweight individuals [58,60-62], a large inter-individual range was noted (MFO range: $26-353 \mathrm{mg} \cdot \mathrm{min}^{-1}$ ). As such, normalised data were also assessed to confirm or support findings. When data were normalised, taking into consideration individual responses to the intervention, MFO, relative MFO and FAT $_{\text {MIN }}$ were all significantly enhanced with dGTE+ overall compared with PL, but not dGTE. However, for steady state exercise, normalised data only confirmed improved substrate utilisation for dGTE+ based on a reduced RER and the contribution of carbohydrate to total EE compared with dGTE. Whilst this further supports the contention that dGTE+ improved fat oxidation dynamics and substrate utilisation efficiency, data should be interpreted with caution based on individual 'responders' to the protocol.

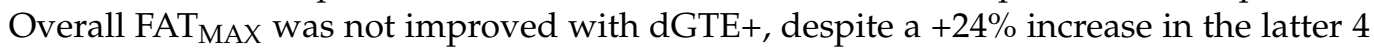
weeks of this study. This may be explained with the wide range of FAT $_{\mathrm{MAX}}$ values observed across this study (range: 24.1 to $52.4 \% \dot{\mathrm{VO}}_{2 \text { peak }}$ ).

Previous studies have reported mixed findings on the effect of GTE on fat oxidation, with several observing improvements in whole-body fat oxidation rates $(+17-35 \%$ improvement employing a dose range of $270-400 \mathrm{mg} \cdot \mathrm{d}^{-1}$ EGCG) $[11,17-19,25]$ and no or small effects elsewhere $\left(<+5-7 \%\right.$ improvement with a dose range of $180-624 \mathrm{mg} \cdot \mathrm{d}^{-1}$ EGCG) $[21-24,26,63]$. Indeed, a recent meta-analysis [64] highlighted a non-significant effect of EGCG supplementation on fat oxidation when compared with placebo (mean difference: $210 \mathrm{mg} \cdot \mathrm{min}^{-1}$ ), although these findings were based on pooled data from only two randomised controlled trials and should be interpreted with caution. Interestingly, beneficial effects of GTE have been mainly observed with healthy, active, untrained or sedentary individuals using acute ( $<2$ days) moderate EGCG dose [11,17-19]. However, within these studies, it is unclear whether enhanced fat oxidation was due to accumulated GTE intake or dose consumed prior to exercise testing considering plasma EGCG concentrations peak at $\sim 90$ mins, with a reported half-life of $\sim 10$ hours $[65,66]$. It is further unclear whether these short-term studies have meaningful impact in the longer term. Chronic intake of dGTE, with comparable EGCG dose, but not consumed on the day of testing, has been shown to enhance fat oxidation and exercise performance in healthy, recreationally active males [25]. However, the use of dGTE has been disputed elsewhere [26] with physically active males, and appears to offer little benefit to trained individuals in terms of substrate utilisation and/or endurance exercise performance [21-24]. 
Collectively, these studies indicate that EGCG (both caffeinated and decaffeinated) may be effective in recreationally active or less trained individuals, potentially via thermogenic mechanisms pertinent to COMT inhibition and increased lipolytic activity leading to enhanced $\mathrm{FAT}_{\mathrm{OX}}$ during exercise $[9,14]$, although supporting evidence to confirm this is currently lacking. In the current study, it is noteworthy that normalised FFAs were greater for both dGTE groups compared with PL, albeit non-significantly. In the longer term, the effectiveness of this mechanism may be related to COMT genotype [67] and/or specific adaptations in mitochondrial efficiency with regular EGCG consumption (particularly when coupled with exercise training). It has been proposed that EGCG consumption may act in a 'calorie-restriction-mimetic' manner through AMPK and sirtuin pathways. This may further modulate PGC1 $\alpha$, peroxisome proliferator-activated receptors (PPARs) and Forkhead box O (FOXO) gene expression, particularly in liver, adipose and skeletal muscle tissue $[14,15,68]$. However, this has been disputed elsewhere in animal studies, with inference that adaptations in fatty acid translocase/CD36mRNA are more likely associated with improvements in fat oxidation [69]. Future studies should aim to explore underlying mechanisms pertinent to fat metabolism gene expression, $\beta$-oxidation enzymes and fatty acid transporters following regular EGCG consumption. Further attention towards EGCG dose, as well as the effects of other potential calorie-restriction-mimetic nutrients (e.g., resveratrol, LA, curcumin, and other plant polyphenols [i.e., gallic acid]) on mitochondrial efficiency are also warranted.

In the current study, the specific use of dGTE did not significantly enhance fat oxidation measures in agreement with previous research [21,22,26,63]. However, the use of a novel dGTE+ formula does support findings particularly pertinent to less trained and/or overweight individuals $[11,17,19]$. The inclusion of quercetin within the dGTE+ may have reduced degradation or oxidation of EGCG within the small intestine (especially considering dietary quercetin intakes were consistent between groups across the intervention). It has been suggested that this could enhance EGCG bioavailability either directly, or through increased EGCG delivery to the large intestine, microbiota conversion to EGCG ring-fission metabolites and phenolic compounds, and subsequent elevated plasma free and conjugated forms of EGCG [31,32,65,70]. Additionally, the increased bioavailability of quercetin, even at a low dose may have supported antioxidant mechanisms of EGCG pertinent to longer-term mitochondria efficiency [70].

The additional inclusion of LA in the dGTE+ formula may have also enhanced EGCG stability through local antioxidant mechanisms, leading to increased EGCG availability. However, as EGCG bioavailability was not ascertained in the current study, this cannot be confirmed. As the use of dGTE only did not significantly influence FAT $_{\mathrm{OX}}$, it is feasible the observed metabolic benefits associated with dGTE+ may be specific to LA. Whilst numerous mechanisms have been proposed, LA supports mitochondrial efficiency as a cofactor for $\alpha$-ketoacid dehydrogenases [71]. Reduced LA (DHLA) has also been implicated as a powerful natural antioxidant, highlighting that LA may support endogenous antioxidant status and mitochondrial protection [71-73]. Additionally, LA has been proposed to influence AMPK pathways which may modulate muscle glucose uptake via GLUT4 translocation, and insulin sensitivity [71,72]. Therefore, in the longer term, it is feasible that, coupled with regular exercise training, LA could support enhanced mitochondrial efficiency, $\mathrm{FAT}_{\mathrm{OX}}$ and subsequent weight loss. However, whilst a recent meta-analysis supports the contention that LA may elicit small, meaningful weight reduction benefits, studies ranging from 8-52 weeks have employed higher LA doses between 300 and $1800 \mathrm{mg} \cdot \mathrm{d}^{-1}$ [74], which may explain such findings. As a lower dose of LA was employed in the current study, it is proposed that the synergistic inclusion of LA and quercetin may have acted to enhance EGCG bioavailability leading to up-regulated fat oxidation pathways in addition to regular exercise. Further research is warranted to confirm EGCG bioavailability with synergistic inclusion of quercetin and/or LA, along with cellular mechanistic pathways, particularly considering higher doses commonly used in commercial formulas. 
Whilst the use of dGTE+ impacted on fat oxidation, by the end of the intervention, neither dGTE supplementation influenced overall body composition measures. This was despite relative, albeit small reductions in overall mean body mass $(-0.50 \mathrm{~kg})$, body fat percentage $(-0.50 \%)$, fat mass $(-0.37 \mathrm{~kg})$, abdominal circumference $(-0.77 \mathrm{~cm})$ or SAD $(-0.61 \mathrm{~cm})$, particularly for dGTE + compared with PL. Furthermore, the relative reduction in estimated VFA with dGTE+ was $~ 3$-fold greater than for both dGTE and PL, albeit non-significant. Maintenance of habitual exercise only marginally impacted on body composition indices as evidenced by a significant reduction in waist and abdomen circumference for PL and dGTE at week 4 within group only. Collectively, the results indicate that when habitual exercise and dietary intake are controlled for, dGTE supplementation did not favour improved body composition indices. As dGTE+ only enhanced FAT $\mathrm{Ox}$ by week 8, any changes in body composition attributed to EGCG mechanisms may require longer interventions based on overall relative patterns in body composition indices with dGTE+. Indeed, a previous meta-analysis [9] highlighted that GT consumption across studies exceeding 12 weeks resulted in a small, positive effect on body mass loss $(-1.31 \mathrm{~kg})$, with greater effects $(-1.60 \mathrm{~kg})$ observed for low habitual caffeine intake users.

However, the lack of efficacy of dGTE to modify body composition measures does concur with other longer-term studies (EGCG dose: $199-843 \mathrm{mg} \cdot \mathrm{d}^{-1}$ ) $[28,67,75]$, although physical activity was only partially monitored in these studies, and one study [28] also introduced a low energy diet as part of the intervention. Elsewhere, significant improvements in body composition have been reported with GTE consumption (EGCG dose: 100-225 mg. $\left.\mathrm{d}^{-1}\right)[12,13,29]$, particularly when combined with an exercise intervention in previously sedentary individuals [12,29]. Interestingly, in one study [29] exercise prescription began at $40 \%$ HR reserve (HRR) and progressed up to 50-59\% HRR. Other studies utilising training at FAT $_{\text {MAX }}$ (range: 34-52\% $\dot{\mathrm{VO}}_{2 \max }[62,76,77]$ ) in overweight, obese or diabetic women demonstrated significant improvements in body mass, BMI, fat mass and visceral trunk fat. In the current study, physical activity guidelines $\left(>150 \mathrm{mins} \cdot \mathrm{wk}^{-1}\right.$ at a moderate intensity $\sim 60-65 \% \dot{\mathrm{VO}}_{2 \text { peak }}$ in line with other studies $\left.[18,78]\right)$ were maintained. As this intensity was above participants' FAT $_{\text {MAX }}$, this likely favoured carbohydrate metabolism and, in part, may explain the small decrease in MFO observed with PL. Combin-

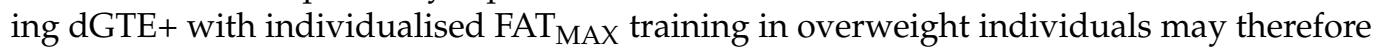
favourably improve body composition, warranting further research.

Cardio-metabolic variables were largely unaffected by either dGTE supplementation, with the exception of a medium effect size reported for LDL-c which was $\sim 11 \%$ lower with $\mathrm{dGTE}+$ by week 8 , and further supported via normalised values. The relative reduction in total LDL-c across the intervention with dGTE $+\left(-0.38 \pm 0.22 \mathrm{mmol} \cdot \mathrm{L}^{-1}\right)$ was comparable to previous studies $[5,79]$, which may be important as the mean values were considered elevated. This concurs with a recent meta-analysis indicating that LDL-c is likely reduced with regular green tea consumption (all sources) by $-0.12 \mathrm{mmol} \cdot \mathrm{L}^{-1}$ [80], and likely further reduced $\left(-0.14 \mathrm{mmol} \cdot \mathrm{L}^{-1}\right)$ when capsulated or decaffeinated green tea products are used [80]. Interestingly, the observed effects of green tea consumption (all sources) appear greater over time, with noted reductions for total cholesterol $\left(-0.14 \mathrm{mmol} \cdot \mathrm{L}^{-1}\right)$ and triglycerides $\left(-0.23 \mathrm{mmol} \cdot \mathrm{L}^{-1}\right)$ in longer-term clinical trials ( $>12$ weeks) [80]. In the current study, as cholesterol biomarkers were largely unaffected with dGTE alone, this infers that the observed reductions in LDL-c with dGTE+ may have been attributed to either increased bioavailability via inclusion of low-dose quercetin, or separately via the inclusion of LA acting independently. Indeed, other reviews [81] have reported significant reductions in LDL-c of $-0.33 \mathrm{mmol} \cdot \mathrm{L}^{-1}$ with LA alone, which is comparable to that observed in the current study.

Both leptin and adiponectin levels were unaffected across the intervention with dGTE or dGTE+. This agrees with a recent meta-analysis [82] demonstrating that leptin concentration is likely unaffected with GT (from all sources) when consumed for less than 12 weeks. In contrast, longer-term use of GT (>12 weeks) likely increases leptin concentrations (effect size: 2.90 [82]). As appetite hormones and body composition changes were largely unaf- 
fected by either dGTE in the current study, this suggests that observed benefits of dGTE+ on fat oxidation by week 8 may have been attributed to gene expression associated with mitochondrial efficiency [14]. It is noteworthy that adiponectin levels were significantly higher with dGTE+ compared to PL at baseline, week 4 and 8. Adiponectin has been associated with synergistic activation of AMPK, p38 mitogen-activated protein kinase (MAPK) and PPAR $\alpha$ in skeletal muscle, and hence fatty acid oxidation [83]. Higher adiponectin levels, combined with dGTE+, may have favourably altered AMPK kinetics, resulting in enhanced mitochondrial fatty acid oxidation across the intervention. In accordance with previous reviews [82], it is feasible that longer-term dGTE consumption further influences appetite regulation favouring improved body composition characteristics, especially when combined with enhanced fat metabolism.

It is also noteworthy, when considering normalised values, that mean insulin, HOMAIR and leptin changes were elevated with PL across the intervention (albeit non-significantly), with negligible changes observed for dGTE or dGTE+. It is therefore unclear whether the small changes observed for PL were related to dietary composition differences, or whether dGTE acted in a 'protective' manner. However, collectively, these results support previous findings that GTE was ineffective in improving cardio-metabolic function $[6,27,28]$, but differ from other studies where positive effects of GTE have been observed with [30] or without exercise [5,13]. Interestingly, one other study [84] employed a theaflavin-enriched GTE strategy, resulting in significant reductions in LDL-c and total cholesterol. This combination was proposed to reduce intestinal absorption and/or increased excretion of cholesterol, as well as LDL-c liver receptor up-regulation. The combined use of quercetin and LA with dGTE + in the current study may have similarly enhanced dGTE effectiveness.

Whilst dGTE+ also resulted in a significant reduction in resting HR by week 4 compared to dGTE, but not by week 8 , this did not impact on blood pressure measurements. This is somewhat surprising as a recent meta-analysis on green tea (from all sources) highlighted studies ( $\leq 12$ weeks) resulting in a reduction of $-1.32 \mathrm{mmHg}$ for SBP (nonsignificant) and $-1.78 \mathrm{mmHg}$ for DBP (deemed significant), with lower observed effects for capsulated green tea, and green tea (all sources) consumed over longer periods ( $>12$ weeks) [85]. In the current study, dGTE+ resulted in a reduction of $-3.33 \mathrm{mmHg}$ (for $\mathrm{SBP}$ ) and $-1.78 \mathrm{mmHg}$ (for DBP), which exceeded the observed reduction expected for exercise/placebo ( $-2.44 \mathrm{mmHg}$ for SBP and $-0.78 \mathrm{mmHg}$ for DBP), but was deemed non-significant. Whilst this may be partly explained by inter-individual variance in blood pressure responses, our results suggest that decaffeinated green tea extract did not impact on blood pressure response above that expected through maintenance of regular exercise.

An important aspect of the current study was the inclusion of liver enzyme and bilirubin measures. This was undertaken based on current concerns with the potential for GTE-induced hepatotoxicity [86]. However, no significant elevations were observed for any measures, indicating relative safety of dGTE at a moderate dose across the intervention period. Whilst it has been noted that fasted intake may be a contributing factor to GTE-induced heptatoxicity [87], other studies have highlighted the potential for reduced bioavailability when GTE is consumed with food [20]. In the current study, participants were requested to consume dGTE away from food in a split-dose manner. As such, it is possible that consumption may have occurred in an absorptive state. Coupled with a lower single dose per consumption (i.e., 200 mg EGCG per capsule), this may, in part, explain normal liver function observed in the current study. Whilst this supports previous research [6], and is in line with current recommendations [87,88], the efficacy of lower doses is warranted, as well as awareness of product composition. As example, unpublished data from our laboratory indicate that exposure to the specific dGTE used in this study impacts AMPK activity and mitochondrial oxidative stress to improve survival of hepatocytes in vitro. Therefore, due consideration of the effect of dGTE on oxidative stress pathways at a cellular level is also needed to understand the molecular mechanisms impacting MFO, as other products may differ in terms of biological effects. 
It is important to note several limitations of the current study. As previously mentioned, EGCG bioavailability was not undertaken, and as no LA-specific intervention was included, findings should be interpreted with caution. Further studies investigating the specific effects of LA with or without dGTE are therefore warranted. In the current study a standardised dGTE (70\% EGCG) product was sourced based on our previous research [25]. Whilst this was independently verified, the content of other catechins (e.g., (-)-epicatechin-3-gallate (ECG)), flavonols (e.g., isoquercitin), flavonol glycosides and their aglycones, were not quantified. Whilst EGCG is the most abundant catechin within dGTE, the remaining catechin/flavonol compounds may have contributed to findings. Consideration to the presence, and biological activity, of other catechins/flavonols within dGTE supplementation is therefore warranted in future research.

In a similar context, consideration to dietary consistency and total polyphenol intake is also warranted. In the present study, relative dietary intake consistency was established in the 7 day period leading into each testing session. Furthermore, estimated quercetin intake (overall mean: $20.6 \pm 8.5 \mathrm{mg} \cdot \mathrm{d}^{-1}$ ) was comparable between groups, and similar to previously reported research $[89,90]$. Therefore, any influence of the dGTE+ compound may have related to the enhanced bioavailability of EGCG with additional quercetin. However, it is feasible that individual dietary variance and total polyphenol intake outside of collection periods may have limited findings. Future research should therefore consider the impact of dGTE strategies in conjunction with low or high dietary polyphenol intake when combined with exercise strategies. Similarly, whilst exercise was monitored across the full intervention period, we did not investigate other components of physical activity such as non-exercise activity thermogenesis or sedentary periods which could affect overall metabolic flexibility and/or fat oxidation, as previously reported [91,92], even when physical activity guidelines are met. Finally, although testing sessions were standardised, as the majority of the participants were females, it is feasible that hormonal variance pertinent to menstrual cycle regulation may have influenced metabolic changes. However, this has been disputed elsewhere [93].

\section{Conclusions}

The use of a novel dGTE+ (containing quercetin and LA) was effective in improving MFO and FAT $\mathrm{MIN}_{\mathrm{N}}$ in healthy, overweight individuals by week 8 of the intervention. This corresponded with improved $\mathrm{FAT}_{\mathrm{OX}}$ during steady state exercise and the reduced contribution of carbohydrate to total energy expenditure. However, when habitual exercise and dietary intake are maintained, this did not translate to improved body composition or markers of cardio-metabolic health, with the exception of reduced LDL-c. Future studies investigating dose-response and longer-term implications of dGTE+, particularly in combination with dietary strategies to support weight-loss in overweight individuals are warranted.

Author Contributions: Conceptualisation, J.D.R., M.B., L.B., L.M. and R.D.; methodology, J.D.R., A.G.B.W., M.B., L.B., L.M. and R.D.; formal analysis, J.D.R., A.G.B.W., M.B., L.M., L.B. and J.D.C.; investigation, H.C. and L.G.; writing-original draft preparation, J.D.R.; writing—review and editing, J.D.R., A.G.B.W., L.B., M.B., R.D., L.M., H.C., L.G., J.D.C.; supervision, J.D.R.; project administration, J.D.R., R.D. and L.M.; funding acquisition, J.D.R. All authors have read and agreed to the published version of this manuscript.

Funding: This research was funded by Biocare Ltd (UK) for consumable and analytical costs only. The APC was funded by Anglia Ruskin University.

Institutional Review Board Statement: The study was conducted according to the guidelines of the Declaration of Helsinki, and approved by the Faculty of Science and Technology Research Ethics Panel, Anglia Ruskin University (approval number: FST/FREP/17/703 on 21/11/17).

Informed Consent Statement: Informed consent was obtained from all subjects involved in the study. 
Data Availability Statement: The data presented in this study are available on request from the corresponding author. The data are not publicly available due to ethical considerations, in accordance with consent provided by participants on the use of confidential data.

Acknowledgments: This study was supported by Biocare Ltd. (UK), with provision of nutritional products.

Conflicts of Interest: The authors declare no conflict of interest. The funders had no role in the design of the study; in the collection, analyses, or interpretation of data; in the writing of the manuscript, or in the decision to publish the results.

\section{References}

1. Henning, S.M.; Niu, Y.; Lee, N.H.; Thames, G.D.; Minuti, R.R.; Wang, H.; Go, V.L.W.; Heber, D. Bioavailability and antioxidant activity of tea flavanols after consumption of green tea, black tea, or a green tea extract supplement. Am. J. Clin. Nutr. 2004, 80, 1558-1564. [CrossRef]

2. Ryu, O.H.; Lee, J.; Lee, K.W.; Kim, H.Y.; Seo, J.A.; Kim, S.G.; Kim, N.H.; Baik, S.H.; Choi, D.S.; Choi, K.M. Effects of green tea consumption on inflammation, insulin resistance and pulse wave velocity in type 2 diabetes patients. Diabetes Res. Clin. Pract. 2006, 71, 356-358. [CrossRef]

3. Liu, L.; Wu, X.; Zhang, B.; Yang, W.; Li, D.; Dong, Y.; Yin, Y.; Chen, Q. Protective effects of tea polyphenols on exhaustive exercise-induced fatigue, inflammation and tissue damage. Food Nutr. Res. 2017, 61, 1333390. [CrossRef]

4. Mukhtar, H.; Ahmad, N. Tea polyphenols: Prevention of cancer and optimizing health. Am. J. Clin. Nutr. 2000, 71, 1698S-1702S. [CrossRef]

5. Nantz, M.P.; Rowe, C.A.; Bukowski, J.F.; Percival, S.S. Standardised capsule of Camellia sinensis lowers cardiovascular risk factors in a randomized, double-blind, placebo-controlled study. Nutrition 2009, 25, 147-154. [CrossRef] [PubMed]

6. Mielgo-Ayuso, J.; Barrenechea, L.; Alcorta, P.; Larrarte, E.; Margareto, J.; Labayen, I. Effects of dietary supplementation with epigallocatechin-3-gallate on weight loss, energy homeostasis, cardiometabolic risk factors and liver function in obese women: Randomised, double-blind, placebo-controlled clinical trial. Br. J. Nutr. 2014, 111, 1263-1271. [CrossRef] [PubMed]

7. Reygaert, W.C. An Update on the Health Benefits of Green Tea. Beverages 2017, 3, 6. [CrossRef]

8. Feng, W.Y. Metabolism of green tea catechins: An overview. Curr. Drug Metab. 2006, 7, 755-809. [CrossRef]

9. Hursel, R.; Viechtbauer, W.; Westerterp-Plantenga, M.S. The effects of green tea on weight loss and weight maintenance: A meta-analysis. Int. J. Obes. 2009, 33, 956-961. [CrossRef]

10. Hursel, R.; Westerterp-Plantenga, M.S. Thermogenic ingredients and body weight regulation. Int. J. Obes. 2010, 34, 659-669. [CrossRef] [PubMed]

11. Dulloo, A.G.; Duret, C.; Rohrer, D.; Girardier, L.; Mensi, N.; Fathi, M.; Chantre, P.; Vandermander, J. Efficacy of a green tea extract rich in catechin polyphenols and caffeine in increasing 24-h energy expenditure and fat oxidation in humans. Am. J. Clin. Nutr. 1999, 70, 1040-1050. [CrossRef]

12. Maki, K.C.; Reeves, M.S.; Farmer, M.; Yasunaga, K.; Matsuo, N.; Katsuragi, Y.; Komikado, M.; Tokimitsu, I.; Wilder, D.; Jones, F.; et al. Green tea catechin consumption enhances exercise-induced abdominal fat loss in overweight and obese adults. J. Nutr. 2009, 139, 264-270. [CrossRef]

13. Nagao, T.; Hase, T.; Tokimitsu, I. A green tea extract high in catechins reduces body fat and cardiovascular risks in humans. Obesity 2007, 15, 1473-1483. [CrossRef]

14. Hodgson, A.B.; Randell, R.K.; Jeukendrup, A.E. The effect of green tea extract on fat oxidation at rest and during exercise: Evidence of efficacy and proposed mechanisms. Adv. Nutr. 2013, 4, 129-140. [CrossRef]

15. Testa, G.; Biasi, F.; Poli, G.; Chiarpotto, E. Calorie restriction and dietary restriction mimetics: A strategy for improving healthy aging and longevity. Curr. Pharm. Des. 2014, 20, 2950-2977. [CrossRef]

16. Ghasemi, E.; Afzalpour, M.E.; Nayebifar, S. Combined high-intensity interval training and green tea supplementation enhance metabolic and antioxidant status in response to acute exercise in overweight women. J. Physiol. Sci. 2020, 70, 31. [CrossRef] [PubMed]

17. Boschmann, M.; Thielecke, F. The effects of epigallocatechin-3-gallate on thermogenesis and fat oxidation in obese men: A pilot study. J. Am. Coll. Nutr. 2007, 26, 389S-395S. [CrossRef] [PubMed]

18. Venables, M.C.; Hulston, C.J.; Cox, H.R.; Jeukendrup, A.E. Green tea extract ingestion, fat oxidation, and glucose tolerance in healthy humans. Am. J. Clin. Nutr. 2008, 87, 778-784. [CrossRef] [PubMed]

19. Gahreman, D.; Wang, R.; Boutcher, Y.; Boutcher, S. Green Tea, Intermittent Sprinting Exercise, and Fat Oxidation. Nutrients 2015, 7, 5646-5663. [CrossRef]

20. Naumovski, N.; Blades, B.L.; Roach, P.D. Food Inhibits the Oral Bioavailability of the Major Green Tea Antioxidant Epigallocatechin Gallate in Humans. Antioxidants 2015, 4, 373-393. [CrossRef] [PubMed]

21. Sugita, M.; Kapoor, M.P.; Nishimura, A.; Okubo, T. Influence of green tea catechins on oxidative stress metabolites at rest and during exercise in healthy humans. Nutrition 2016, 32, 321-331. [CrossRef] [PubMed]

22. Dean, S.; Braakhuis, A.; Paton, C. The effects of EGCG on fat oxidation and endurance performance in male cyclists. Int. J. Sport Nutr. Exerc. Metab. 2009, 19, 624-644. [CrossRef] 
23. Eichenberger, P.; Colombani, P.C.; Mettler, S. Effects of 3-week consumption of green tea extracts on whole-body metabolism during cycling exercise in endurance-trained men. Int. J. Vitam. Nutr. Res. 2009, 79, 24-33. [CrossRef] [PubMed]

24. Eichenberger, P.; Mettler, S.; Arnold, M.; Colombani, P.C. No effects of three-week consumption of a green tea extract on time trial performance in endurance-trained men. Int. J. Vitam. Nutr. Res. 2010, 80, 54-64. [CrossRef] [PubMed]

25. Roberts, J.D.; Roberts, M.G.; Tarpey, M.D.; Weekes, J.C.; Thomas, C.H. The effect of a decaffeinated green tea extract formula on fat oxidation, body composition and exercise performance. J. Int. Soc. Sports Nutr. 2015, 12, 1. [CrossRef]

26. Randell, R.K.; Hodgson, A.B.; Lotito, S.B.; Jacobs, D.M.; Rowson, M.; Mela, D.J.; Jeukendrup, A.E. Variable Duration of Decaffeinated Green Tea Extract Ingestion on Exercise Metabolism. Med. Sci. Sports Exerc. 2014, 46, 1185-1193. [CrossRef]

27. Amozadeh, H.; Shabani, R.; Nazari, M. The Effect of Aerobic Training and Green Tea Supplementation on Cardio Metabolic Risk Factors in Overweight and Obese Females: A Randomized Trial. Int. J. Endocrinol. Metab. 2018, 16, e60738. [CrossRef]

28. Diepvens, K.; Kovacs, E.M.; Nijs, I.M.; Vogels, N.; Westerterp-Plantenga, M.S. Effect of green tea on resting energy expenditure and substrate oxidation during weight loss in overweight females. Br. J. Nutr. 2005, 94, 1026-1034. [CrossRef]

29. Bagheri, R.; Rashidlamir, A.; Ashtary-Larky, D.; Wong, A.; Alipour, M.; Motevalli, M.S.; Chebbi, A.; Laher, I.; Zouhal, H. Does green tea extract enhance the anti-inflammatory effects of exercise on fat loss? Br. J. Clin. Pharmacol. 2020, 86, 753-762. [CrossRef]

30. Bagheri, R.; Rashidlamir, A.; Ashtary-Larky, D.; Wong, A.; Grubbs, B.; Motevalli, M.S.; Baker, J.S.; Laher, I.; Zouhal, H. Effects of green tea extract supplementation and endurance training on irisin, pro-inflammatory cytokines, and adiponectin concentrations in overweight middle-aged men. Eur. J. Appl. Physiol. 2020, 120, 915-923. [CrossRef]

31. Wang, P.; Heber, D.; Henning, S.M. Quercetin increased bioavailability and decreased methylation of green tea polyphenols in vitro and in vivo. Food Funct. 2012, 3, 635-642. [CrossRef]

32. Kale, A.; Gawande, S.; Kotwal, S.; Netke, S.; Roomi, W.; Ivanov, V.; Niedzwiecki, A.; Rath, M. Studies on the effects of oral administration of nutrient mixture, quercetin and red onions on the bioavailability of epigallocatechin gallate from green tea extract. Phytother. Res. 2010, 24 (Suppl. 1), S48-S55. [CrossRef]

33. Chen, W.L.; Kang, C.H.; Wang, S.G.; Lee, H.M. $\alpha$-Lipoic acid regulates lipid metabolism through induction of sirtuin 1 (SIRT1) and activation of AMP-activated protein kinase. Diabetologia 2012, 55, 1824-1835. [CrossRef]

34. Pandit, A.P.; Joshi, S.R.; Dalal, P.S.; Patole, V.C. Curcumin as a permeability enhancer enhanced the antihyperlipidemic activity of dietary green tea extract. BMC Complement. Altern. Med. 2019, 19, 129. [CrossRef]

35. Moon, Y.J.; Morris, M.E. Pharmacokinetics and bioavailability of the bioflavonoid biochanin A: Effects of quercetin and EGCG on biochanin A disposition in rats. Mol. Pharm. 2007, 4, 865-872. [CrossRef]

36. El-Kholy, W.M.; El-Said, F.G.; Mousa, A.T. The Role of Green Tea And Alpha-Lipoic Acid In Preventing Some Risks Of Rheumatoid Arthritis In Female Rats. Egypt. J. Exp. Biol. (Zoo.) 2007, 3, 1-8.

37. Narotzki, B.; Reznick, A.Z.; Navot-Mintzer, D.; Dagan, B.; Levy, Y. Green tea and vitamin E enhance exercise-induced benefits in body composition, glucose homeostasis, and antioxidant status in elderly men and women. J. Am. Coll. Nutr. 2013, 32, 31-40. [CrossRef]

38. Faul, F.; Erdfelder, E.; Lang, A.-G.; Buchner, A. G*power 3: A flexible statistical power analysis program for the social, behavioral, and biomedical sciences. Behav. Res. Methods 2007, 39, 175-191. [CrossRef] [PubMed]

39. Riebe, D. (Ed.) ACSM's Guidelines for Exercise Testing and Prescription, 10th ed.; Lippincott Williams and Wilkins: Philadelphia, PA, USA, 2017; pp. 69-79, ISBN-10: 9781496339072.

40. Pintér, Z.; Pósa, A.; Varga, C.; Horváth, I.; Palkó, A.; Just, A.; Pálfi, G. Anthropometric dimensions provide reliable estimates of abdominal adiposity: A validation study. J. Comp. Hum. Biol. 2017, 68, 398-409. [CrossRef]

41. Achten, J.; Jeukendrup, A.E. Maximal fat oxidation during exercise in trained men. Int. J. Sports Med. 2003, 24, 603-608. [CrossRef]

42. Dandanell, S.; Husted, K.; Amdisen, S.; Vigelsø, A.; Dela, F.; Larsen, S.; Helge, J.W. Influence of maximal fat oxidation on long-term weight loss maintenance in humans. J. Appl. Physiol. 2017, 123, 267-274. [CrossRef]

43. Borg, G. Ratings of perceived exertion and heart rates during short-term cycle exercise and their use in a new cycling strength test. Int. J. Sports Med. 1982, 3, 153-158. [CrossRef] [PubMed]

44. Jeukendrup, A.E.; Wallis, G.A. Measurement of substrate oxidation during exercise by means of gas exchange measurements. Int. J. Sports Med. 2005, 26, S28-S37. [CrossRef] [PubMed]

45. Zakrzewski, J.; Tolfrey, K. Exercise protocols to estimate Fatmax and maximal fat oxidation in children. Pediatr. Exerc. Sci. 2011, 23, 122-135. [CrossRef]

46. Antonio, J.; Peacock, C.; Ellerbroek, A.; Fromhoff, B.; Silver, T. The effects of consuming a high protein diet (4.4 g/kg/d) on body composition in resistance-trained individuals. J. Int. Soc. Sports Nutr. 2014, 11, 19. [CrossRef] [PubMed]

47. Antonio, J.; Ellerbroek, A.; Silver, T.; Orris, S.; Scheiner, M.; Gonzalez, A.; Peacock, C. A high protein diet (3.4 g/kg/d) combined with a heavy resistance training program improves body composition in healthy trained men and women-A follow-up investigation. J. Int. Soc. Sports Nutr. 2015, 12, 39. [CrossRef]

48. Comyns, T.; Flanagan, E.P. Applications of the session rating of perceived exertion system in professional rugby union. Strength Cond. J. 2013, 35, 78-85. [CrossRef]

49. Foster, C.; Florhaug, J.A.; Franklin, J.; Gottschall, L.; Hrovatin, L.A.; Parker, S.; Doleshal, P.; Dodge, C. A new approach to monitoring exercise training. J Strength Cond. Res. 2001, 15, 109-115. [PubMed] 
50. Schulz, J.M.; Birmingham, T.B.; Atkinson, H.F.; Woehrle, E.; Primeau, C.A.; Lukacs, M.J. Are we missing the target? Are we aiming too low? What are the aerobic exercise prescriptions and their effects on markers of cardiovascular health and systemic inflammation in patients with knee osteoarthritis? A systematic review and meta-analysis. Br. J. Sports Med. 2020, 54, 771-775. [CrossRef] [PubMed]

51. Friedewald, W.T.; Levy, R.I.; Fredrickson, D.S. Estimation of the concentration of low-density lipoprotein cholesterol in plasma, without use of the preparative ultracentrifuge. Clin. Chem. 1972, 18, 499-502. [CrossRef]

52. Levy, J.C.; Matthews, D.R.; Hermans, M.P. Correct homeostasis model assessment (HOMA) evaluation uses the computer program. Diabetes Care 1998, 21, 2191-2192. [CrossRef]

53. Amaro-Gahete, F.J.; Sanchez-Delgado, G.; Jurado-Fasoli, L.; De-la-O, A.; Castillo, M.J.; Helge, J.W.; Ruiz, J.R. Assessment of maximal fat oxidation during exercise: A systematic review. Scand. J. Med. Sci. Sports 2019, 29, 910-921. [CrossRef]

54. Amaro-Gahete, F.J.; Ruiz, J.R. Methodological issues related to maximal fat oxidation rate during exercise: Comment on: Change in maximal fat oxidation in response to different regimes of periodized high-intensity interval training (HIIT). Eur. J. Appl. Physiol. 2018, 118, 2029-2031. [CrossRef]

55. Chrzanowski-Smith, O.J.; Edinburgh, R.M.; Thomas, M.P.; Haralabidis, N.; Williams, S.; Betts, J.A.; Gonzalez, J.T. The day-to-day reliability of peak fat oxidation and FAT MAX. Eur. J. Appl. Physiol. 2020, 120, 1745-1759. [CrossRef] [PubMed]

56. Abildgaard, J.; Pedersen, A.T.; Green, C.J.; Harder-Lauridsen, N.M.; Solomon, T.P.; Thomsen, C.; Pedersen, M.; Pedersen, J.T.; Mortensen, O.H.; Pilegaard, H.; et al. Menopause is associated with decreased whole body fat oxidation during exercise. Am. J. Physiol. Endocrinol. Metab. 2013, 304, E1227-E1236. [CrossRef]

57. Chenevière, X.; Malatesta, D.; Peters, E.M.; Borrani, F. A mathematical model to describe fat oxidation kinetics during graded exercise. Med. Sci. Sports Exerc. 2009, 41, 1615-1625. [CrossRef] [PubMed]

58. Croci, I.; Hickman, I.J.; Wood, R.E.; Borrani, F.; Macdonald, G.A.; Byrne, N.M. Fat oxidation over a range of exercise intensities: Fitness versus fatness. Appl. Physiol. Nutr. Metab. 2014, 39, 1352-1359. [CrossRef] [PubMed]

59. Del Coso, J.; Hamouti, N.; Ortega, J.F.; Mora-Rodriguez, R. Aerobic fitness determines whole-body fat oxidation rate during exercise in the heat. Appl. Physiol. Nutr. Metab. 2010, 35, 741-748. [CrossRef]

60. Besnier, F.; Lenclume, V.; Gérardin, P.; Fianu, A.; Martinez, J.; Naty, N.; Porcherat, S.; Boussaid, K.; Schneebeli, S.; Jarlet, E.; et al. Individualized exercise training at maximal fat oxidation combined with fruit and vegetable-rich diet in overweight or obese women: The LIPOXmax-Réunion randomized controlled trial. PLoS ONE 2015, 10, e0139246. [CrossRef]

61. Tsujimoto, T.; Sasai, H.; Miyashita, M.; Eto, M.; So, R.; Ohkubo, H.; Tanaka, K. Effect of weight loss on maximal fat oxidation rate in obese men. Obes. Res. Clin. Pract. 2012, 6, e91-e174. [CrossRef]

62. Wang, J.; Tan, S.; Cao, L. Exercise training at the maximal fat oxidation intensity improved health-related physical fitness in overweight middle-aged women. J. Exerc. Sci. Fit. 2015, 13, 111-116. [CrossRef]

63. Nieman, D.C.; Simonson, A.; Sakaguchi, C.A.; Sha, W.; Blevins, T.; Hattabaugh, J.; Kohlmeier, M. Acute Ingestion of a Mixed Flavonoid and Caffeine Supplement Increases Energy Expenditure and Fat Oxidation in Adult Women: A Randomized, Crossover Clinical Trial. Nutrients 2019, 11, 2665. [CrossRef] [PubMed]

64. Kapoor, M.P.; Sugita, M.; Fukuzawa, Y.; Okubo, T. Physiological effects of epigallocatechin-3-gallate (EGCG) on energy expenditure for prospective fat oxidation in humans: A systematic review and meta-analysis. J. Nutr. Biochem. 2017, 43, 1-10. [CrossRef]

65. Fernández, V.A.; Toledano, L.A.; Lozano, N.P.; Tapia, E.N.; Roig, M.D.G.; De la Torre Fornell, R.; García Algar, Ó. Bioavailability of Epigallocatechin Gallate Administered With Different Nutritional Strategies in Healthy Volunteers. Antioxidants 2020, 9, 440. [CrossRef]

66. Chow, H.H.; Cai, Y.; Alberts, D.S.; Hakim, I.; Dorr, R.; Shahi, F.; Crowell, J.A.; Yang, C.S.; Hara, Y. Phase I pharmacokinetic study of tea polyphenols following single-dose administration of epigallocatechin gallate and polyphenon E. Cancer Epidemiol. Biomark. Prev. 2001, 10, 53-58.

67. Dostal, A.M.; Samavat, H.; Espejo, L.; Arikawa, A.Y.; Stendell-Hollis, N.R.; Kurzer, M.S. Green Tea Extract and Catechol-OMethyltransferase Genotype Modify Fasting Serum Insulin and Plasma Adiponectin Concentrations in a Randomized Controlled Trial of Overweight and Obese Postmenopausal Women. J. Nutr. 2016, 146, 38-45. [CrossRef]

68. Rupasinghe, H.P.; Sekhon-Loodu, S.; Mantso, T.; Panayiotidis, M.I. Phytochemicals in regulating fatty acid $\beta$-oxidation: Potential underlying mechanisms and their involvement in obesity and weight loss. Pharmacol. Ther. 2016, 165, 153-163. [CrossRef]

69. Murase, T.; Haramizu, S.; Shimotoyodome, A.; Nagasawa, A.; Tokimitsu, I. Green tea extract improves endurance capacity and increases muscle lipid oxidation in mice. Am. J. Physiol. Regul. Integr. Comp. Physiol. 2005, 288, R708-R715. [CrossRef] [PubMed]

70. Egert, S.; Wolffram, S.; Bosy-Westphal, A.; Boesch-Saadatmandi, C.; Wagner, A.E.; Frank, J.; Rimbach, G.; Mueller, M.J. Daily quercetin supplementation dose-dependently increases plasma quercetin concentrations in healthy humans. J. Nutr. 2008, 138, 1615-1621. [CrossRef] [PubMed]

71. Shay, K.P.; Moreau, R.F.; Smith, E.J.; Smith, A.R.; Hagen, T.M. Alpha-lipoic acid as a dietary supplement: Molecular mechanisms and therapeutic potential. Biochim. Biophys. Acta 2009, 1790, 1149-1160. [CrossRef] [PubMed]

72. Salehi, B.; Yılmaz, Y.B.; Antika, G.; Tumer, T.B.; Mahomoodally, M.F.; Lobine, D.; Akram, M.; Riaz, M.; Capanoglu, E.; Sharopov, F.; et al. Insights on the Use of $\alpha$-Lipoic Acid for Therapeutic Purposes. Biomolecules 2019, 9, 356. [CrossRef] [PubMed]

73. McCarty, M.F.; Barroso-Aranda, J.; Contreras, F. The 'rejuvenatory' impact of lipoic acid on mitochondrial function in aging rates may reflect induction and activation of PPAR $\gamma$-coactivator-1 $\alpha$. Med. Hypotheses 2009, 72, 29-33. [CrossRef] 
74. Kucukgoncu, S.; Zhou, E.; Lucas, K.B.; Tek, C. Alpha-lipoic acid (ALA) as a supplementation for weight loss: Results from a meta-analysis of randomized controlled trials. Obes. Rev. 2017, 18, 594-601. [CrossRef] [PubMed]

75. Janssens, P.L.; Hursel, R.; Westerterp-Plantenga, M.S. Long-term green tea extract supplementation does not affect fat absorption, resting energy expenditure, and body composition in adults. J. Nutr. 2015, 145, 864-870. [CrossRef] [PubMed]

76. Cao, L.; Jiang, Y.; Li, Q.; Wang, J.; Tan, S. Exercise Training at Maximal Fat Oxidation Intensity for Overweight or Obese Older Women: A Randomized Study. J. Sports Sci. Med. 2019, 18, 413-418. [PubMed]

77. Tan, S.; Du, P.; Zhao, W.; Pang, J.; Wang, J. Exercise Training at Maximal Fat Oxidation Intensity for Older Women with Type 2 Diabetes. Int. J. Sports Med. 2018, 39, 374-381. [CrossRef]

78. Ichinose, T.; Nomura, S.; Someya, Y.; Akimoto, S.; Tachiyashiki, K.; Imaizumi, K. Effect of endurance training supplemented with green tea extract on substrate metabolism during exercise in humans. Scand. J. Med. Sci. Sports 2011, 21, 598-605. [CrossRef]

79. Huang, L.H.; Liu, C.Y.; Wang, L.Y.; Huang, C.J.; Hsu, C.H. Effects of green tea extract on overweight and obese women with high levels of low density-lipoprotein-cholesterol (LDL-C): A randomised, double-blind, and cross-over placebo-controlled clinical trial. BMC Complement. Altern. Med. 2018, 18, 294. [CrossRef]

80. Xu, R.; Yang, K.; Li, S.; Dai, M.; Chen, G. Effect of green tea consumption on blood lipids: A systematic review and meta-analysis of randomized controlled trials. Nutr. J. 2020, 19, 48. [CrossRef]

81. Mousavi, S.M.; Shab-Bidar, S.; Kord-Varkaneh, H.; Khorshidi, M.; Djafarian, K. Effect of alpha-lipoic acid supplementation on lipid profile: A systematic review and meta-analysis of controlled clinical trials. Nutrition 2019, 59, 121-130. [CrossRef]

82. Haghighatdoost, F.; Nobakht, M.; Gh, B.F.; Hariri, M. Effect of green tea on plasma leptin and ghrelin levels: A systematic review and meta-analysis of randomized controlled clinical trials. Nutrition 2018, 45, 17-23. [CrossRef] [PubMed]

83. Yoon, M.J.; Lee, G.Y.; Chung, J.J.; Ahn, Y.H.; Hong, S.H.; Kim, J.B. Adiponectin increases fatty acid oxidation in skeletal muscle cells by sequential activation of AMP-activated protein kinase, p38 mitogen-activated protein kinase, and peroxisome proliferator-activated receptor alpha. Diabetes 2006, 55, 2562-2570. [CrossRef] [PubMed]

84. Maron, D.J.; Lu, G.P.; Cai, N.S.; Wu, Z.G.; Li, Y.H.; Chen, H.; Zhu, J.Q.; Jin, X.J.; Wouters, B.C.; Zhao, J. Cholesterol-Lowering Effect of a Theaflavin-Enriched Green Tea Extract: A Randomized Controlled Trial. Arch. Intern. Med. 2003, 163, 1448-1453. [CrossRef]

85. Xu, R.; Yang, K.; Ding, J.; Chen, G. Effect of green tea supplementation on blood pressure: A systematic review and meta-analysis of randomized controlled trials. Medicine 2020, 99, e19047. [CrossRef]

86. Mazzanti, G.; Di Sotto, A.; Vitalone, A. Hepatotoxicity of green tea: An update. Arch. Toxicol. 2015, 89, 1175-1191. [CrossRef]

87. Hu, J.; Webster, D.; Cao, J.; Shao, A. The safety of green tea and green tea extract consumption in adults-Results of a systematic review. Regul. Toxicol. Pharmacol. 2018, 95, 412-433. [CrossRef]

88. Dekant, W.; Fujii, K.; Shibata, E.; Morita, O.; Shimotoyodome, A. Safety assessment of green tea based beverages and dried green tea extracts as nutritional supplements. Toxicol. Lett. 2017, 277, 104-108. [CrossRef]

89. Li, Y.; Yao, J.; Han, C.; Yang, J.; Chaudhry, M.T.; Wang, S.; Liu, H.; Yin, Y. Quercetin, Inflammation and Immunity. Nutrients 2016, 8, 167. [CrossRef] [PubMed]

90. Ranka, S.; Gee, J.; Biro, L.; Brett, G.; Saha, S.; Kroon, P.; Skinner, J.; Hart, A.R.; Cassidy, A.; Rhodes, M.; et al. Development of a food frequency questionnaire for the assessment of quercetin and naringenin intake. Eur. J. Clin. Nutr. 2008, 62, 1131-1138. [CrossRef] [PubMed]

91. Rynders, C.A.; Blanc, S.; DeJong, N.; Bessesen, D.H.; Bergouignan, A. Sedentary behaviour is a key determinant of metabolic inflexibility. J. Physiol. 2018, 596, 1319-1330. [CrossRef]

92. Amaro-Gahete, F.J.; Acosta, F.M.; Migueles, J.H.; Ponce González, J.G.; Ruiz, J.R. Association of sedentary and physical activity time with maximal fat oxidation during exercise in sedentary adults. Scand. J. Med. Sci. Sports 2020, 30, 1605-1614. [CrossRef] [PubMed]

93. Frandsen, J.; Pistoljevic, N.; Quesada, J.P.; Amaro-Gahete, F.J.; Ritz, C.; Larsen, S.; Dela, F.; Helge, J.W. Menstrual cycle phase does not affect whole body peak fat oxidation rate during a graded exercise test. J. Appl. Physiol. 2020, 128, 681-687. [CrossRef] [PubMed] 